\title{
CONTRIBUTION OF IDAHO NATIONAL LABORATORY TO THE CRP FUMAC
}

GIOVANNI PASTORE, RICHARD WILLIAMSON, Jason Hales, Kyle Gamble, Russell Gardner, James Tompkins

February 2018

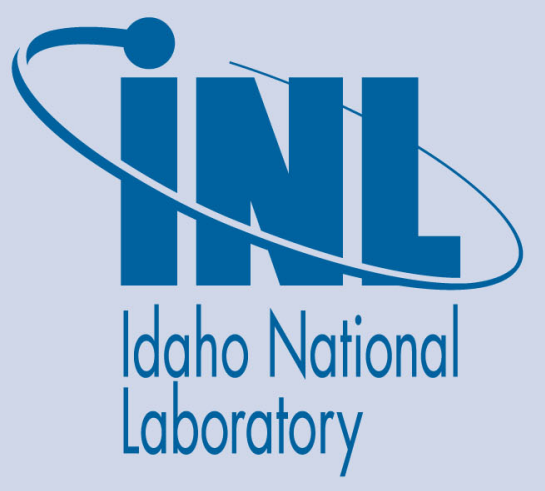

The INL is a U.S. Department of Energy National Laboratory operated by Battelle Energy Alliance 


\title{
CONTRIBUTION OF IDAHO NATIONAL LABORATORY TO THE CRP FUMAC
}

\author{
GIOVANNI PASTORE, RICHARD WILLIAMSON, Jason Hales, Kyle Gamble, \\ Russell Gardner, James Tompkins
}

February 2018

Idaho National Laboratory Idaho Falls, Idaho 83415

http://www.inl.gov

Prepared for the

U.S. Department of Energy

Under DOE Idaho Operations Office

Contract DE-AC07-05ID14517 


\title{
CONTRIBUTION OF IDAHO NATIONAL LABORATORY TO THE CRP FUMAC
}

\author{
GIOVANNI PASTORE, RICHARD WILLIAMSON, JASON HALES, KYLE GAMBLE, \\ RUSSELL GARDNER, JAMES TOMPKINS
}

\section{Fuel Modeling and Simulation Department, Idaho National Laboratory, Idaho Falls, USA}

\begin{abstract}
This report summarizes the contribution of Idaho National laboratory (INL) to the IAEA Coordinated Research Project on Fuel Modeling under Accident Conditions FUMAC. In line with the original research agreement between INL/US DOE/Battelle and IAEA, work at INL has focused on both (i) development of INL's fuel performance code BISON for the analysis of loss-of-coolant accidents (LOCA) and (ii) simulation of selected FUMAC priority cases. With reference to code development efforts, models were implemented in BISON for high temperature cladding oxidation, Zircaloy solid-solid phase transformation, Zircaloy high temperature creep, cladding burst failure and axial fuel relocation. BISON, analyses were performed of the FUMAC cases (1) MTA EK tests PUZRY, (2) QUENCH L1 rods 4 and 7, (3) Halden IFA-650.2 and (4) Halden IFA-650.10. In addition, the REBEKA separate effects tests were analyzed, including an effort to investigate 3D cladding response in presence of azimuthal temperature variations. In general, BISON predictions of burst temperature, pressure and time to burst are very reasonable. Predictions of cladding hoop strain are less satisfactory and require additional investigation. Finally, results of 3D simulations indicated that $3 \mathrm{D}$ effects are potentially important in fuel rod analysis during LOCAs. BISON results are made available to the FUMAC project as a contribution to the FUMAC benchmark exercise.
\end{abstract}

\section{INTRODUCTION}

To coordinate and support research on nuclear fuel modeling under accident conditions in member countries following the Fukushima accident, the International Atomic Energy Agency (IAEA) sponsored the Coordinated Research Project (CRP) on Fuel Modeling under Accident Conditions (FUMAC).

The US Department of Energy (DOE) has been developing state-of-the-art capabilities to simulate of nuclear fuel behavior within the Nuclear Energy Advanced Modeling and Simulation (NEAMS) and Consortium for Advanced Simulation of Light Water Reactors (CASL) programs. The result is the BISON code [1], a multidimensional, finite-element based fuel performance code developed at Idaho National Laboratory (INL). Validation work for BISON has focused initially on Light Water Reactor (LWR) fuel during normal operating conditions and power ramps [2]. More recently, significant work has been performed on BISON development and validation for the analysis of accident scenarios such as loss of coolant accidents (LOCA) [3,4] and Reactivity Insertion Accidents (RIA) [5,6].

This report gives an account of INL's accomplishments in the framework of the CRP FUMAC. The proposal of INL for participation in FUMAC [7] included

- Development of the BISON fuel performance code to include models for phenomena relevant to fuel rod behavior during LOCAs.

- Simulation of some of the FUMAC cases using BISON, with results being made available to the project.

The work has been performed along the lines outlined in the proposal, with the BISON code having been extended to simulation of LOCA accident scenarios and applied to the analysis of 
several FUMAC cases. In this report we provide a description of BISON developments for LOCA analysis carried out throughout the project and present and discuss the BISON simulations. As agreed upon during the First Research Coordination Meeting (RCM1), the FUMAC cases that have been analyzed with BISON are (1) MTA EK tests PUZRY, (2) QUENCH L1 rods 4 and 7, (3) Halden IFA-650.2, and (4) Halden IFA-650.10. In addition, simulations of the ballooning tests REBEKA were performed and are included in this report, in view of the potential interest to the FUMAC project. This additional work included 3D simulations accounting for azimuthal temperature variations.

The work on BISON development and validation for LOCAs, including INL's contribution to FUMAC, has benefited from collaboration between INL and the Halden Reactor Project. This collaboration has been strengthened by having BISON developers working onsite in Norway.

The structure of this report is as follows. In Section 2 we summarize BISON enhancements for the analysis of LOCA behavior. In Section 3 we present BISON simulations of the FUMAC cases, as well as additional calculations of potential interest to the project. For each of the considered cases, we include a description of the experiment, details of the BISON setup for the simulations, and a report and discussion of the results. Results are made available to FUMAC for the benchmark exercise. A final chapter provides conclusions and recommendations from the INL activities within FUMAC.

\section{BISON DEVELOPMENTS FOR LOCA ANALYSIS}

From the beginning, BISON has incorporated a large-strain mechanics formulation, essential to correctly analyze cladding ballooning during LOCAs. In order to capture the complex material response during accident situations, however, it is also necessary to incorporate in the code specific models dealing with the high-temperature, transient phenomena involved. For this purpose, dedicated material models have been incorporated in the thermo-mechanics analysis framework of BISON. Models are overviewed in this section.

BISON's capability enhancements for accident analysis performed during this work and applied to the simulations presented in Section 2 include models for high-temperature steam oxidation of Zircaloy cladding, crystallographic phase transformation of Zircaloy, hightemperature cladding creep and cladding failure due to burst [3-4].

In addition, BISON's model of fission gas swelling and release in $\mathrm{UO}_{2}$ was extended to include a specific treatment of the burst release effect during transients. This transient model was originally developed based on power ramp data [8,9] and has not been re-calibrated and validated yet for LOCA transients. However, it has been applied with some success to RIA design basis accident simulations [6], and can potentially be adapted and applied to the improved simulation of FGR during LOCA transients. In view of its potential for the modeling of fission gas behavior during DBAs, this development is deemed relevant to the FUMAC project and has been included in this report. The development of the transient fission gas behavior model was carried out in collaboration with POLIMI (Italy) and JRC-Karlsruhe (European Commission, Germany).

Also, two recent BISON developments for LOCA analysis, i.e., cladding oxidation energy deposition and axial fuel relocation, have not yet been applied to the simulations described in Section 3. However, these are new BISON capabilities that are in place and relevant to LOCA analysis, thus they are deemed of potential interest to the FUMAC project and included in this report as an additional contribution. These capabilities will be applied in future LOCA simulations with BISON. 


\subsection{High-temperature cladding oxidation}

The process of oxidation of Zircaloy through an exothermic reaction with the coolant affects both thermal and mechanical performance of the cladding. In the high temperature range (e.g., LOCA) the coolant has become steam, and oxidation proceeds much more rapidly than at normal LWR operating temperatures. Under these conditions, the kinetics of oxide scale growth and oxygen mass gain can be described by a parabolic law, with the reaction rate constant defined as a function of the temperature through an Arrhenius relation [10]:

$\frac{d \xi}{d t}=A \cdot \exp \left(-\frac{Q}{R T_{I}}\right)$

where $\xi$ is either the oxide scale thickness, $\xi=s(\mathrm{~m})$, or the oxygen mass, $\xi=g\left(\mathrm{~kg} \cdot \mathrm{m}^{-2}\right), t$ (s) the time, $A\left(\mathrm{~m}\right.$ or $\left.\mathrm{kg} \cdot \mathrm{m}^{-2}\right)$ the pre-exponential factor, $Q(\mathrm{~J} / \mathrm{mol})$ the activation energy, $R$ $(\mathrm{J} / \mathrm{mol}-\mathrm{K})$ the universal gas constant, and $T_{I}(\mathrm{~K})$ the metal-oxide interface temperature. Following the recommendations in [10], the BISON model includes correlations for oxide scale growth and oxygen mass gain rates in Zircaloy-2/4 appropriate to different temperature ranges. In particular, the following approach is adopted:

- For metal-oxide interface temperatures from $673 \mathrm{~K}$ up to $1800 \mathrm{~K}$, the Leistikov [11] correlation is used. The Cathcart-Pawel correlation [12] is also available and can be chosen as an option. The Leistikov correlation has been selected as reference in view of the larger underlying database, the availability of experimentally determined mass gain for all tests, and the better fit for lower temperature relative to the Cathcart-Pawel correlation [10].

- Above $1900 \mathrm{~K}$, the Prater-Courtright correlation [13] is used.

- Between 1800 and $1900 \mathrm{~K}$, a linear interpolation is made. Linear interpolation between two correlations of Arrhenius type is obtained by a third correlation of the same type [10].

The values of the parameters in Eq. 2.1 relative to the different correlations are given in Table 2.1 .

\subsection{Phase transformation of the cladding material}

An increase in the cladding temperature above $\sim 1000-1100 \mathrm{~K}$, which may occur during a LOCA, involves time and temperature dependent phase transformation of the $\mathrm{Zr}$ alloy from hexagonal ( $\alpha$-phase) to cubic ( $\beta$-phase) crystal structure. Modeling the kinetics of crystallographic phase transformation is needed for the assessment of the mechanical properties essential for fuel rod integrity (deformation and burst) during a postulated LOCA.

Table 2.1. Parameters of the correlations for oxide scale (S) and oxygen mass gain (g) at high temperature [10].

\begin{tabular}{lllll}
\hline Correlation & $A_{S}\left(\mathrm{~m}^{2} \mathrm{~s}^{-1}\right)$ & $Q_{S} / R(\mathrm{~K})$ & $A_{g}\left(\mathrm{~kg} \cdot \mathrm{m}^{-2}\right)$ & $Q_{g} / R(\mathrm{~K})$ \\
\hline Leistikov & $7.82 \cdot 10^{-6}$ & 20214 & 52.42 & 20962 \\
Cathcart-Pawel & $2.25 \cdot 10^{-6}$ & 18062 & 36.22 & 20100 \\
Prater-Courtright & $2.98 \cdot 10^{-3}$ & 28420 & $3.3 \cdot 10^{3}$ & 26440 \\
\hline
\end{tabular}


The crucial parameter for the transformation kinetics is the evolution of the volume fraction of the new phase as a function of time and temperature. A model has been implemented in BISON for calculation of the volume fraction of $\beta$-phase in Zircaloy-4 as a function of time and temperature during phase transformation in non-isothermal conditions. The model is based on [14-16]. The phase transformation rate is expressed by

$\frac{d y}{d t}=k(T)\left[y_{s}(T)-y\right]$

where $y$ is the volume fraction of $\beta$-phase, $y_{s}$ (/) the steady-state or equilibrium value of $y$, and $k\left(\mathrm{~s}^{-1}\right)$ the rate parameter. The $\beta$-phase equilibrium fraction is a sigmoid function of temperature

$y_{s}=\frac{1}{2}\left[1+\tanh \left(\frac{T-T_{\text {cent }}}{T_{\text {span }}}\right)\right]$

where $T_{\text {cent }}$ and $T_{\text {span }}$ are material specific parameters related to the center and span of the mixed-phase temperature region, respectively. For Zircaloy-4, $T_{\text {cent }}=1159-0.096 \mathrm{w}(\mathrm{K})$ and $T_{\text {span }}=44+0.026 \mathrm{w}(\mathrm{K})[14]$ are used, with $w$ being the hydrogen concentration in the range $0 \leq w \leq 1000$ wppm (weight parts per million hydrogen). The rate parameter is expressed in the form

$k=k_{0} \exp \left(-\frac{E}{k_{b} T(t)}\right)+k_{m}$

where $k_{0}$ is a kinetic factor, $E$ an effective activation energy, $k_{b}$ the Boltzmann constant, and $k_{m}$ a constant. For Zircaloy-4, $k_{0}=60457+18129|q|\left(\mathrm{s}^{-1}\right)$ and $E / k_{b}=16650(\mathrm{~K})[14,16]$ are used, where $q=d T / d t\left(\mathrm{Ks}^{-1}\right)$ is the heat rate in the range $0.1 \leq|q| \leq 100 \mathrm{Ks}^{-1}$. The $\alpha \rightarrow \beta$ transformation is purely diffusion controlled, while the $\beta \rightarrow \alpha$ transformation is partly martensitic. This is represented by the constant $k_{m}$ given in the form [16]

$\begin{cases}k_{m}=0 & \alpha \rightarrow \beta \\ k_{m}=0.2 & \beta \rightarrow \alpha\end{cases}$

The starting temperatures for the onset of $\alpha \rightarrow \alpha+\beta$ and $\beta \rightarrow \alpha+\beta$ phase transformations are calculated as (in kelvin) [14]

$$
\begin{aligned}
T_{\alpha \rightarrow \alpha+\beta} & =\left\{\begin{array}{cl}
1083-0.152 w & \text { for } 0 \leq q<0.1 K s^{-1} \\
(1113-0.156 w) Q^{0.0118} & \text { for } 0.1 \leq q \leq 100 K s^{-1}
\end{array}\right. \\
T_{\beta \rightarrow \alpha+\beta} & =\left\{\begin{array}{cc}
1300 & \text { for }-0.1<q \leq 0 \mathrm{Ks}^{-1} \\
1302.8-8.333|Q|^{0.477} & \text { for }-100 \leq q \leq-0.1 K s^{-1}
\end{array}\right.
\end{aligned}
$$

for $0 \leq w \leq 1000$ wppm. Note that $w=0$ is considered in BISON at this time.

The $\beta$-phase volume fraction as a function of time is calculated by numerical integration of Eq. 2.2. The calculated volume fractions of $\beta$ phase as a function of temperature at equilibrium and for temperature variation rates of $\pm 10 \mathrm{Ks}^{-1}$ are shown in Fig. 2.1. 


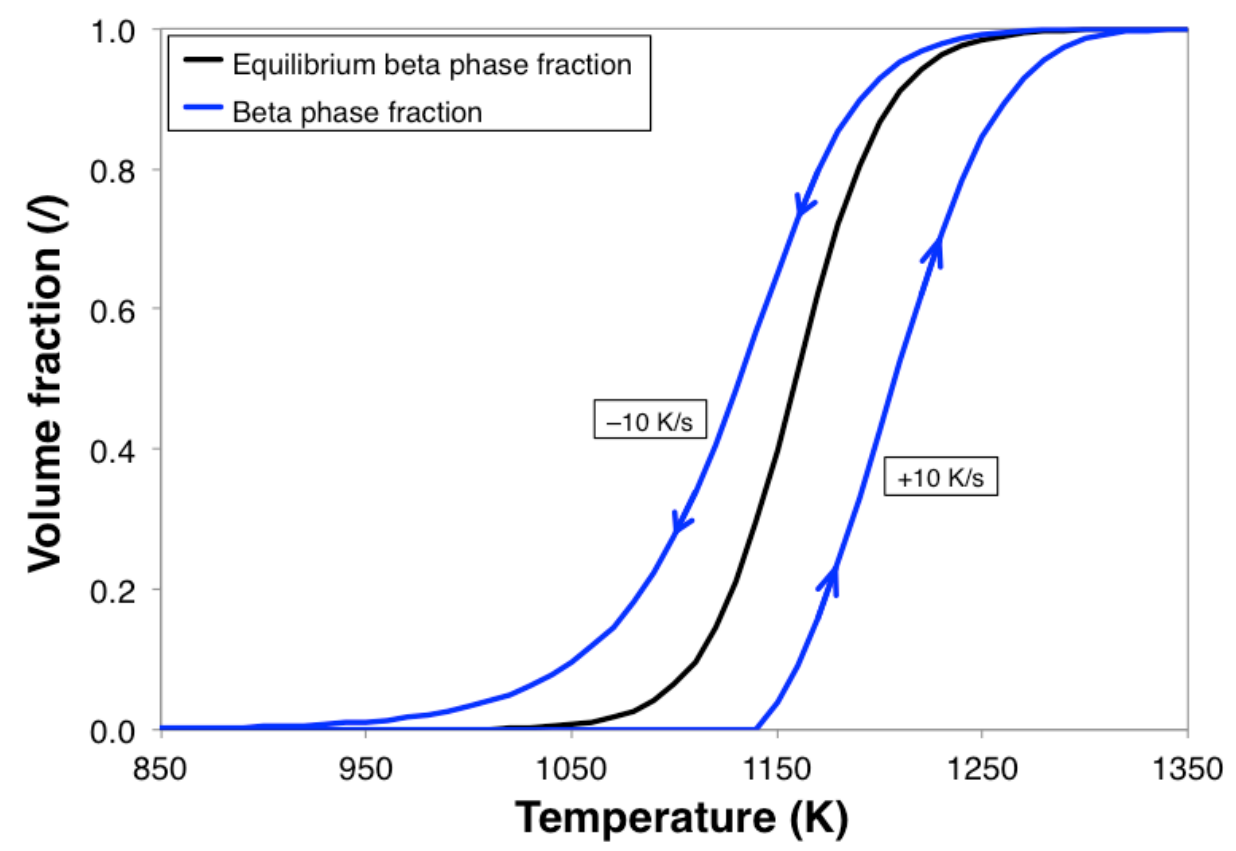

Fig. 2.1. Calculated volume fraction of $\beta$-phase as a function of temperature. Equilibrium conditions (slow temperature variation) and temperature variation rates of $\pm 10 \mathrm{Ks}^{-1}$ are considered.

\subsection{High-temperature creep of Zircaloy cladding}

During a LOCA, outward creep deformation of the cladding tube under the effect of internal pressure and high temperature drives cladding ballooning and eventual failure due to burst. For LOCA analysis, the large creep deformation of the cladding is defined by a strain rate correlation in the form of a Norton power equation [17-19]:

$\dot{\varepsilon}_{e f f}=A \exp \left(-\frac{E_{a}}{R T}\right) \sigma_{e f f}^{n}$

where $\dot{\varepsilon}_{\text {eff }}\left(\mathrm{s}^{-1}\right)$ is the effective creep strain rate, $A\left(\mathrm{MPa}^{-\mathrm{n}} \mathrm{s}^{-1}\right)$ the strength coefficient, $E_{a}\left(\mathrm{~J} \mathrm{~mol}^{-1}\right)$ the activation energy for the creep deformation, $T(\mathrm{~K})$ the temperature, $\sigma_{\text {eff }}(\mathrm{MPa})$ the effective (Von Mises) stress, and $n(-)$ the stress exponent. The material parameters (Table 2.2) used in the model were obtained from tension tests on Zircaloy-4 tubes [18-19]. In the mixed phase $(\alpha+\beta)$ region (Section 2.2), interpolations are made to calculate the Norton parameters. Depending on the strain rate, different approaches are adopted [18]:

Table 2.2. Material parameters used to calculate creep of Zircaloy-4 [19,20]. LI stands for linear interpolation.

\begin{tabular}{lllll}
\hline Phase & $\dot{\varepsilon}_{\text {eff }}\left(\mathrm{s}^{-1}\right)$ & $A\left(\mathrm{MPa}^{\left.-\mathrm{n} \mathrm{s}^{-1}\right)}\right.$ & $E_{a}\left(\mathrm{~J} \mathrm{~mol}^{-1}\right)$ & $n(-)$ \\
\hline$\alpha$ & any & 8737 & $3.21 \cdot 10^{5}+24.69(T-923.15)$ & 5.89 \\
$50 \% \alpha-50 \% \beta$ & $\leq 3 \cdot 10^{-3}$ & 0.24 & 102366 & 2.33 \\
$\beta$ & $>3 \cdot 10^{-3}$ & LI of $\ln (A)$ & LI & LI \\
$\beta$ & any & 7.9 & 141919 & 3.78 \\
\hline
\end{tabular}


- For $\dot{\varepsilon}_{\text {eff }} \leq 3 \cdot 10^{-3} \mathrm{~s}^{-1}$, linear interpolation of $\ln (A), n$ and $E_{a}$ is made between the values for pure $\alpha$ and middle of $\alpha+\beta(50 \% \alpha-50 \% \beta)$ phase, and between $50 \% \alpha-$ $50 \% \beta$ and pure $\beta$ phase.

- For $\dot{\varepsilon}_{\text {eff }}>3 \cdot 10^{-3} \mathrm{~s}^{-1}$, it is assumed that the values of $\ln (A), n$ and $E_{a}$ vary linearly between the values for pure $\alpha$ and pure $\beta$ phase.

To perform the interpolation, the fraction of each phase calculated from a dedicated model as described in Section 2.2Error! Reference source not found. is used.

\subsection{Cladding burst failure model}

For determining the conditions for failure due to burst of Zircaloy-4 cladding during LOCA accidents, the following criteria have been implemented in BISON:

1. An overstress criterion, which assumes that the time of burst is reached when the local hoop stress equals a limiting burst stress [19]:

$\sigma_{\vartheta} \geq \sigma_{b}$

where $\sigma_{\vartheta}$ is the hoop stress and $\sigma_{\mathrm{b}}$ is the burst stress. The burst stress is calculated following Erbacher et al. [19] and depends on the temperature and oxygen concentration. Details can be found in [19] and are not given here for brevity.

2. A plastic instability criterion, which considers cladding burst at the attainment of a limiting value for the effective plastic strain rate:

$\dot{\varepsilon}_{p l, e f f} \geq \dot{\varepsilon}_{b}$

where $\dot{\varepsilon}_{p l, e f f}$ is the effective plastic (creep + plasticity) strain rate and $\dot{\varepsilon}_{b}$ is the limiting value. Following [21], in BISON we use $\dot{\varepsilon}_{b}=100 \mathrm{~h}^{-1} \cong 2.78 \cdot 10^{-2} \mathrm{~s}^{-1}$.

3. A combination of the above criteria, which establishes that cladding burst occurs when either condition 1.9 or 1.10 is fulfilled.

As the overstress criterion may lead to unsafe predictions in low-stress situations [21], either the plastic instability or the combined criterion are used in our calculations (Section 3 ).

\subsection{Transient fission gas behavior in oxide fuel}

Fission gas release (FGR) and gaseous swelling in $\mathrm{UO}_{2}$ fuel are computed in BISON by a physically based model from [22,23]. This model has been recently extended to allow for the rapid FGR (burst release) during transients [8,9]. This new transient capability was originally developed and validated based on annealing and power ramp tests, but can be potentially adapted and applied to DBA transient analysis. Initial application to RIA calculations has led to promising results [6].

In this model, burst release is interpreted as driven by fuel micro-cracking, which is associated with gas depletion of the cracked grain faces during transients and with a corresponding increase in FGR. Gas depletion of a fraction of the grain faces is modeled as a reduction of the fractional grain-face bubble coverage, $F$. In particular, $F$ is scaled by a factor, $f$, corresponding to the fraction of non-cracked (intact) grain faces. The reduction of the 
fractional coverage effectively leads to a decrease of the amount of gas retained in the fuel consequently, of fission gas swelling - and to a corresponding increase of FGR.

We simplify the micro-cracking process into a temperature-dependent behavior, characterized by a micro-cracking parameter, $m$. We also observe that the process can only affect intact grain faces, and write

$\left[\frac{d f}{d t}\right]_{c}=-\frac{d m}{d t} f$

Based on the available experimental evidence, the functional form of $m$ is chosen as a temperature-dependent sigmoid function

$m(T)=1-\left[1+D \exp \left(s \frac{T-T_{\text {infl }}}{B}\right)\right]^{-\frac{1}{D}}$

where $T_{\text {infl }}(\mathrm{K})$ is the value for the temperature at the inflection point of the function $m(T)$ (inflection temperature, or temperature of maximum micro-cracking rate), and $B(\mathrm{~K})$ and $D(-$ ) are parameters related to the temperature-domain width of the phenomenon and the deviation from symmetric behavior during heating/cooling transients, respectively. The value of $s(-)$ is set to +1 during heating transients and to -1 during cooling transients, so that $m$ increases during both heating and cooling. Combination of Eqs. 2.11 and 2.12 leads to a FGR contribution that activates only during temperature variations (transients). In particular, the FGR during a temperature transient will result from the time integral of Eq. 2.11 during the transient.

Model's characteristics are based on the available experimental evidence of transient FGR (e.g., [24-27]). The inflection temperature corresponds to what has been observed experimentally as a critical temperature for the onset of burst release, and $T_{\text {infl }} \sim 1773 \mathrm{~K}$ above 20-30 GWd/tU burnup (e.g., [27]). Rather than adopting a discrete temperature threshold for the onset of burst release, we interpret the observations as a continuous but peaked microcracking (hence, gas release) rate as a function of temperature. Burnup dependence of microcracking, and micro-crack healing, are also accounted for, although details are not given here for brevity. A more extensive description of the model is given in $[8,9]$.

\subsection{Cladding oxidation energy deposition}

During a LOCA the exothermic zirconium oxidation reaction in high temperature steam may lead to significant heat deposition in the cladding. This section describes the addition of an oxidation energy deposition model to capture this effect in BISON.

The conversion of zirconium to zirconium oxide follows the following simple chemical equation [28]:

$2 \mathrm{H}_{2} \mathrm{O}+\mathrm{Zr} \rightarrow 2 \mathrm{H}_{2}+\mathrm{ZrO}_{2}+\mathrm{En}$

The model uses the incremental oxide layer thickness calculated in BISON (Section 2.1) to calculate the energy added to the cladding. In particular, the energy from the zirconium oxidation reaction is calculated as [28]:

$P=\left(\frac{0.74}{0.26}\right)\left(\frac{\Delta W}{\Delta t}\right)\left(2 \pi R_{0}\right) 6.45 \cdot 10^{6}$ 
where $P\left(\mathrm{Wm}^{-1}\right)$ is the linear power from the oxidation reaction, $\Delta W\left(\mathrm{~kg} \cdot \mathrm{m}^{-2}\right)$ the mass gain per unit surface due to oxidation during the time step $\Delta t(\mathrm{~s}), R_{0}(\mathrm{~m})$ the initial cladding outer radius, $6.45 \cdot 10^{6}\left(\mathrm{~J}^{\mathrm{kg}} \mathrm{gg}^{-1}\right)$ the heat of reaction, and $0.74 / 0.26$ is the ratio of $\mathrm{Zr}$ reacted to $\mathrm{O}$ added:

$\frac{\Delta Z r}{\Delta W}=\frac{1-0.26}{0.26}=\frac{0.74}{0.26}$

where $\Delta Z r\left(\mathrm{~kg} \cdot \mathrm{m}^{-2}\right)$ is the mass of zirconium per unit surface area consumed by oxidation during a given time increment, and 0.26 is the weight fraction of oxygen in $\mathrm{ZrO}_{2}$. Eq. 2.15 underlies the assumption that all oxygen forms stoichiometric zirconium oxide.

\subsection{Axial fuel relocation}

Axial relocation of fuel fragments during a LOCA is a phenomenon that causes redistribution of heat within the rod potentially accelerating cladding failure. As the cladding balloons, fragmented and pulverized fuel pellets can fall from upper regions of the rod into the ballooned region. The reduced thermal conductivity of the crumbled fuel and plenum gas mixture, in addition to the increased heat load due to a larger mass of fuel in the ballooned region, results in higher cladding temperatures further exacerbating the cladding distention. The ability to model this complex phenomenon using fuel performance codes is of great importance to ensure accurate predictions of cladding temperature, cladding strain, and the mass of fuel available for dispersal.

Recently, an empirical model was added to BISON to account for the axial relocation phenomenon during LOCAs. In particular, the model developed by Jernkvist and Massih [29] has been incorporated into BISON. The BISON implementation includes (i) the fuel fragmentation and pulverization model to quantify the number and size of fuel fragments and pulvers, the mass fractions of both fragments and pulvers, and an effective packing fraction of the fuel particles, (ii) the axial mass redistribution of the fuel, (iii) the thermal conductivity of the crumbled fuel, and (iv) the radial heat transfer in the fuel rod in presence of crumbled fuel and axial fuel relocation. Details of this model can be found in [29], and are not given here for brevity. The current implementation is based on the 1.5-dimensional capability of BISON. Plans are in place to extend modeling of axial relocation to 2D and 3D calculations.

Jernkvist and Massih [29] propose two test cases denoted as single balloon and twin balloon. These test cases were analyzed with BISON in order to verify the implementation of the axial relocation model. The single balloon verification test is to simulate cladding distention that is maximum at the midplane of the active length. The twin balloon verification test is to simulate the effect of having a spacer-grid at the midplane of the active length. The BISON results of these test cases are compared to the digitized results from the Jernkvist and Massih's report [29] in Fig. 2.2. Three panels are shown for each case representing different times through the duration of the simulation corresponding to 40,60, and $100 \mathrm{~s}$ (final time). The plots show the mass fraction of fuel as a function of axial position. A mass fraction $>1$ indicates that mass has accumulated in this region and a mass fraction $<1$ corresponds to a region partially (or completely) void of fuel. As expected, in the regions near the maximum cladding ballooning the mass fractions are largest. It should be noted that at the very top of the fuel rod the mass fraction remains as 1 . This is because the cladding distention is not large enough in this top layer to allow fuel to relocate out of it. As is evident in both test cases, the BISON implementation has been verified to be correct as the results match Jernkvist and Massih's results extremely well. This model will allow first BISON simulations of LOCA experiments that exhibited significant axial fuel relocation, such as the Halden IFA-650.9 test considered in FUMAC. 

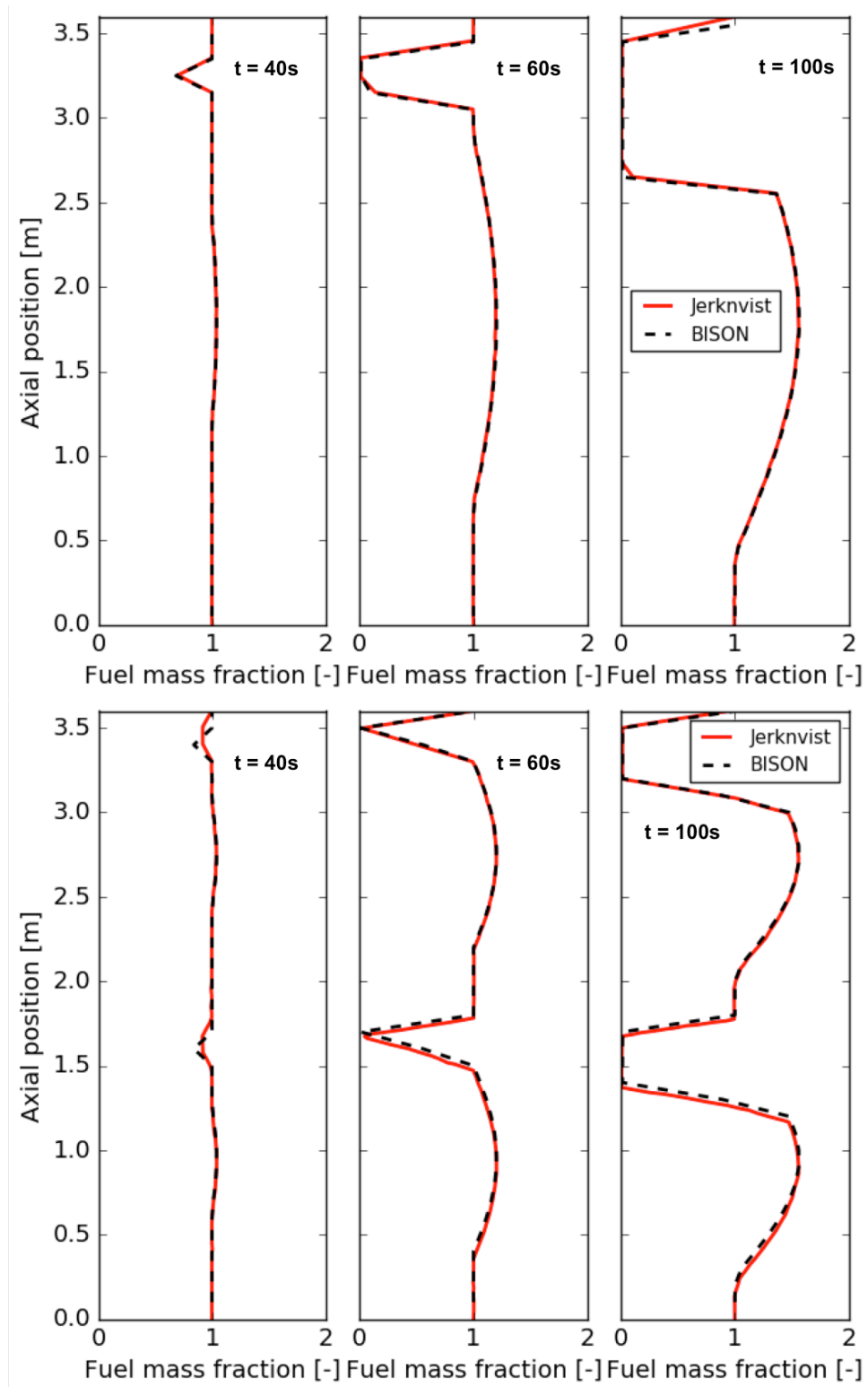

Fig 2.2. Mass fraction as a function of axial position at 40, 60, and $100 \mathrm{~s}$ for single balloon (top) and twin balloon (bottom) test cases. Jernkvist data obtained from [29]. 



\section{BISON SIMULATIONS OF LOCA EXPERIMENTS}

The BISON code, extended with the modeling capabilities described in Section 2, was applied to simulations of LOCA experiments, including several FUMAC priority cases. In particular, BISON analyses for the following cases are presented in this chapter:

- MTA EK separate effects tests PUZRY

- Separate effects tests REBEKA

- QUENCH L1 rods 4 and 7

- Halden test IFA-650.2

- Halden test IFA-650.10

These are all of the cases agreed upon by INL during the FUMAC RCM1. Although the REBEKA tests were not included in FUMAC, they were analyzed as part of the LOCA validation database for BISON. They are included in this report as part of the INL contribution to FUMAC for completeness and also, for one of the REBEKA cases the 3D capability of BISON was applied to investigate 3D cladding response in presence of azimuthal temperature variations. This produced initial insights into 3D effects during LOCAs, which can be of interest to FUMAC, as discussed during the RCM2 [30]. 3D BISON simulations are presented in Section 3.2.

The case EON - Segment 2 was also modeled with BISON [36,31]. However, because this case was later excluded from the FUMAC project, the relative BISON results are not included in this report. More recently, other LOCA cases (not included in FUMAC) were analyzed with BISON. These include the Hardy separate effects experiments [32] and the NRU-MT4 and MT6A fuel rod tests $[33,34]$. These simulations are also not included in this report. Details can be found in [35].

In the following sections, the analyzed experiments are described and the results of the BISON simulations are presented and discussed.

\subsection{MTA EK separate effects tests PUZRY}

INL considered the PUZRY experimental series of isothermal ballooning tests on Zircaloy-4 claddings [36,37]. All of the 31 PUZRY cases were modeled with BISON, although only 6 cases were selected for FUMAC. In this section, we present the overall results for the full set of 31 cases for completeness. We also provide detailed tabulated results for the 6 FUMAC cases.

\section{Description of the tests}

The PUZRY experimental series [36,37] was performed in order to study the mechanical behavior (ballooning and burst) of Zircaloy-4 cladding subject to inner pressure transients at high temperature. In particular, the effects of temperature and pressurization rate on the deformation and the failure (burst) pressure were investigated.

The samples were tested in a resistance furnace providing isothermal conditions in the temperature range of $700-1200^{\circ} \mathrm{C}$. The inner pressure of the test tube was increased linearly until the burst of the sample. After an approximately $1000 \mathrm{~s}$ heat-up period the sample was pressurized with $\mathrm{Ar}$ gas at a constant pressure increase rate. Table 3.1 summarizes temperature and pressure conditions for the PUZRY cases selected for FUMAC. The specimens 
Table 3.1. Conditions of the 6 MTA EK PUZRY cases selected for FUMAC [36,37].

\begin{tabular}{lll}
\hline Test number & Temperature $(\mathrm{K})$ & Pressure ramp rate $(\mathrm{MPa} / \mathrm{s})$ \\
\hline 8 & 1274.15 & 0.00763 \\
10 & 1375.75 & 0.00710 \\
12 & 1470.85 & 0.00723 \\
18 & 1173.35 & 0.01151 \\
26 & 971.55 & 0.01193 \\
30 & 1073.55 & 0.02630 \\
\hline
\end{tabular}

were $50 \mathrm{~mm}$ long Zircaloy-4 tubes. The specimens' inner / outer diameters of 9.3 / $10.75 \mathrm{~mm}$ corresponded to the parameters of PWR fuel cladding. Since the experiments were performed in Ar, cladding corrosion was not investigated.

\section{Setup of the BISON simulations}

Details of the BISON setup adopted for the calculations are as follows.

- Finite-element 2D axisymmetric models of the cladding tubes were used.

- Taking advantage of the symmetry of the problem, only the lower half of the heated cladding length was modeled.

- End plugs were considered by preventing radial motion (i.e., applying zero radial displacement boundary conditions) to the tube inner surfaces in correspondence of the plugs. These correspond to the $5 \mathrm{~mm}$ end sections of the cladding.

- Time-dependent pressures were simulated by Dirichlet pressure boundary conditions applied to the tube inner and outer walls.

- The furnace heating was simulated by a Dirichlet temperature boundary condition applied to the tube outer wall. In the PUZRY database, tubes temperature profiles along the axial direction are not given. However, applying a perfectly uniform temperature axially would lead to a distributed ballooning along the tube, while several experiments showed localized ballooning with maximum strain and burst occurring near the tube's mid-plane. This can be interpreted as associated with axial temperature variations that, albeit small, lead to significant strain axial variations by virtue of the strong (exponential) temperature dependence of the creep rate (see Section 2.3). To account for this, we included a slight axial temperature variation in the BISON simulations. Within FUMAC, Katalin Kulacsy communicated that axial temperature variations of 5-6 K along the central $50 \mathrm{~mm}$ section of the furnace can be expected, based on measurements performed in another furnace [38]. On this base, in the BISON simulations we applied a linear temperature profile (simplest possibility in absence of detailed indications) with the maximum temperature applied at the midplane. The overall (tube end to tube mid-plane) variation was made equal to $6 \mathrm{~K}$, with the average (tube quarter-length) temperature being equal to the experimental value.

- Prior to the pressure transient, we considered the initial heat-up period by applying atmospheric $(0.1 \mathrm{MPa})$ pressure to both sides of the tube and ramping the temperature up from ambient $(300 \mathrm{~K})$ to the test temperature over $1000 \mathrm{~s}$. The inner pressure transient from $0.1 \mathrm{MPa}$ at the experimental rate was applied afterwards, under isothermal conditions. Outer tube pressure was kept constant at $0.1 \mathrm{MPa}$. 
- The combined overstress and plastic instability criterion for cladding burst failure (Section 2.4) was used.

\section{Results}

We present comparisons of the BISON simulation results for the 31 PUZRY cases to the experimental data. Figures 3.1 and 3.2 show the comparisons of cladding inner pressure at cladding burst and time to burst, respectively. Note that the axes in these plots have a logarithmic scale. The accuracy of BISON predictions appears reasonable.

We also present the BISON results in terms of maximum engineering hoop strain at the cladding outer surface at the time of burst. Comparisons of calculated strains to experimental data for the 31 PUZRY cases are shown (on a linear scale) in Figure 3.3. Predictions deviate from experimental data by up to a factor of $\sim 2.6$. Average deviation is of a factor of $\sim 1.7$. Prediction of cladding strains is notoriously difficult for fuel performance codes, even more for LOCA calculations whereby very high strain rates are reached as cladding burst is approached. This implies that the maximum strain reached in the calculation is very sensitive to the specific criterion adopted to determine the time of rod burst (thus, the final time of the calculation and the time at which strain is considered), since small differences in the final time may correspond to large differences in the maximum strain. This has been clearly demonstrated by a previous study by JRC-Karlsruhe where different failure criteria were tested in cladding ballooning and bust simulations with the TRANSURANUS code [21].

Further investigation and sensitivity analysis of the dependence of calculated strains upon the choice of the burst criterion (e.g., in line with [21]) and the relative uncertainties is of interest in perspective. Besides this, further developments of the cladding creep model (e.g., considering anisotropic creep) may also improve strain predictions.

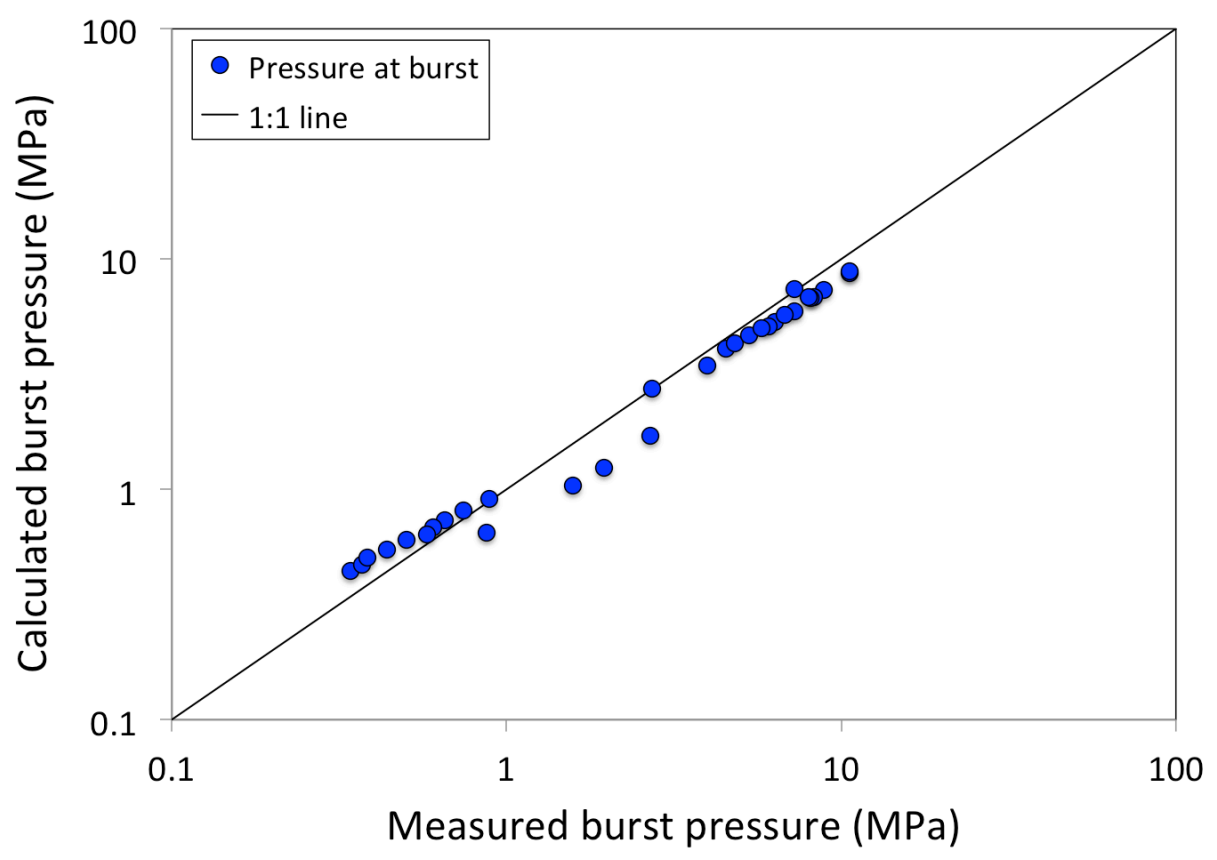

Fig 3.1. Comparison of calculated and measured tube inner pressures at burst for the PUZRY cases. 


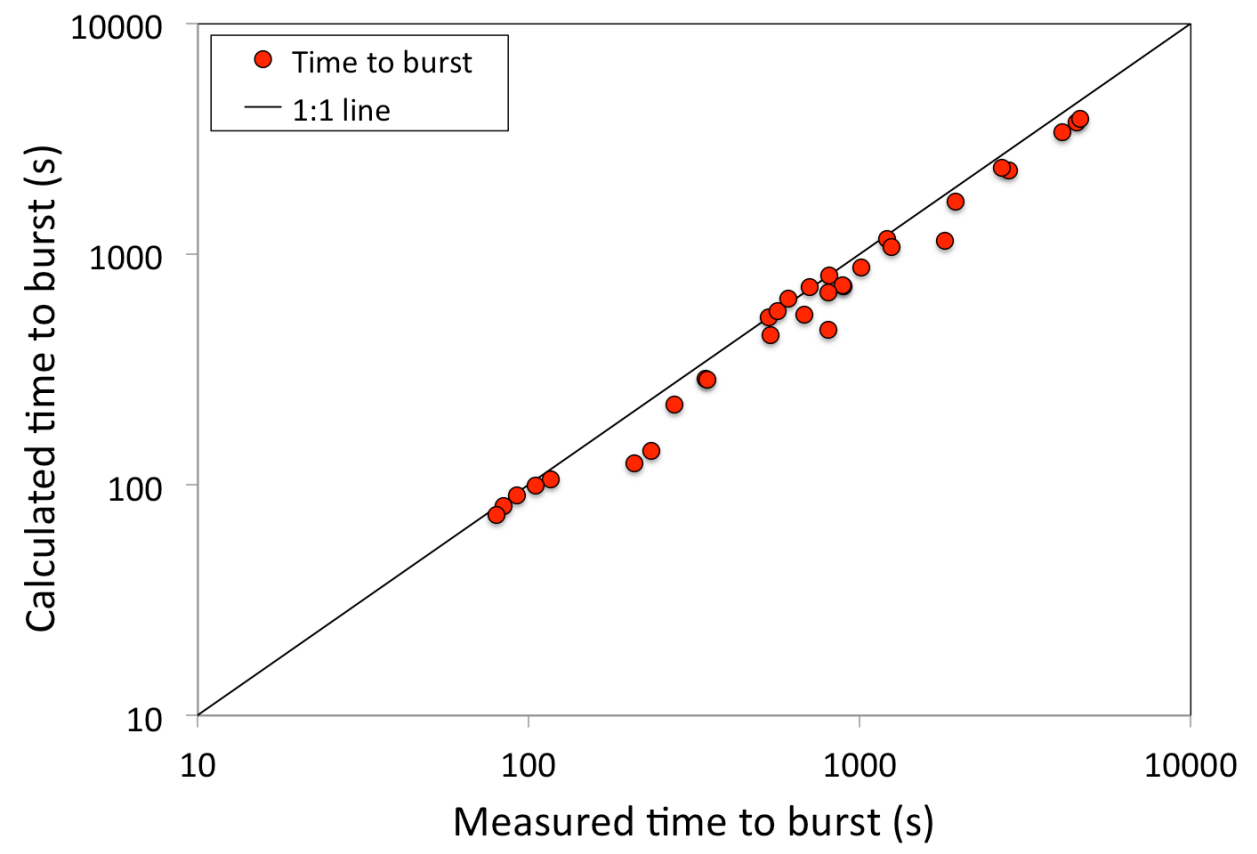

Fig 3.2. Comparison of calculated and measured time to burst for the PUZRY cases.

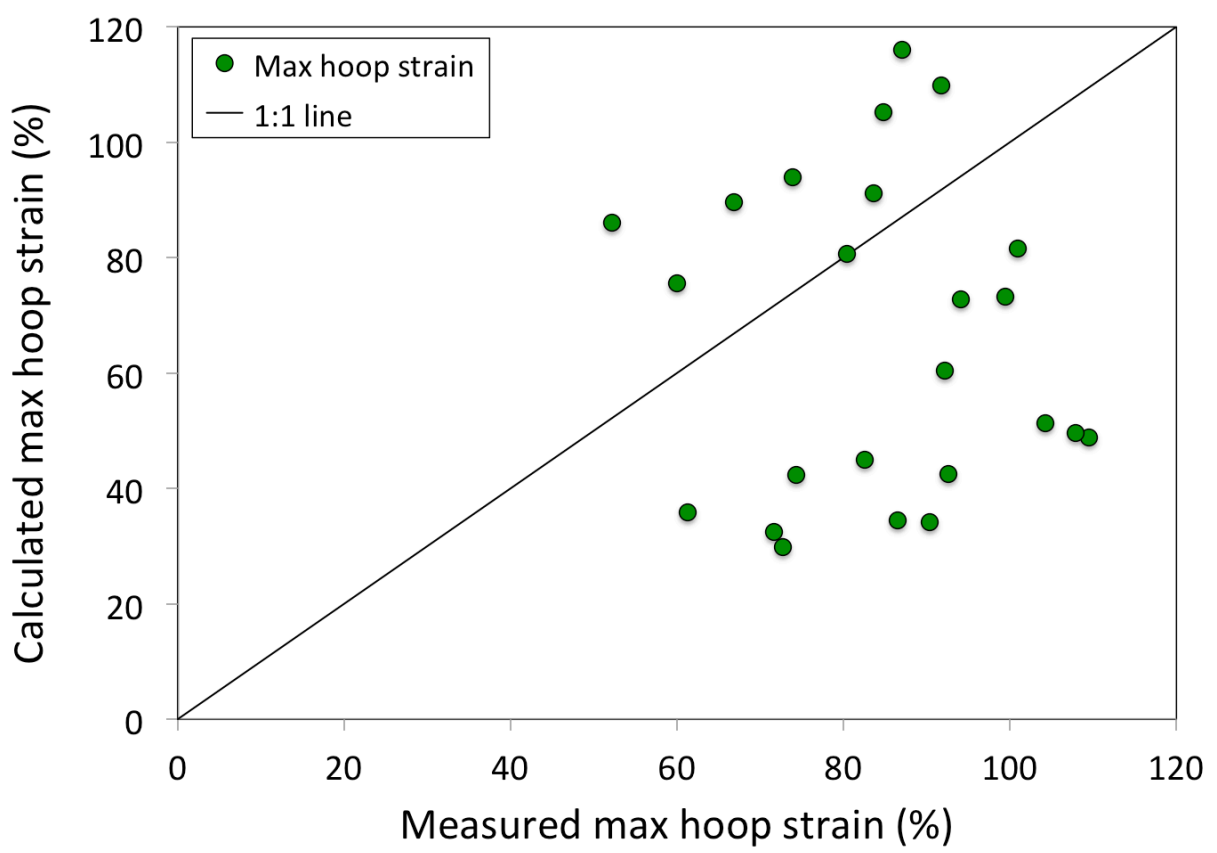

Figure 3.3. Comparison of calculated and measured maximum engineering hoop strain at the cladding outer surface at the time of burst for the PUZRY cases.

Calculated and experimental burst times are plotted as a function of test temperature in Fig. 3.4. The reduction of the burst time as a function of the temperature is reproduced. Deviations of predictions from the experimental data appear to increase at the lower test temperatures.

In Fig. 3.5 and 3.6 we present comparisons between BISON predictions and experimental data of burst pressure and time to burst as a function of test temperature, for the 6 FUMAC cases only. Again, accuracy is very good for the higher temperature cases and grows worse at 


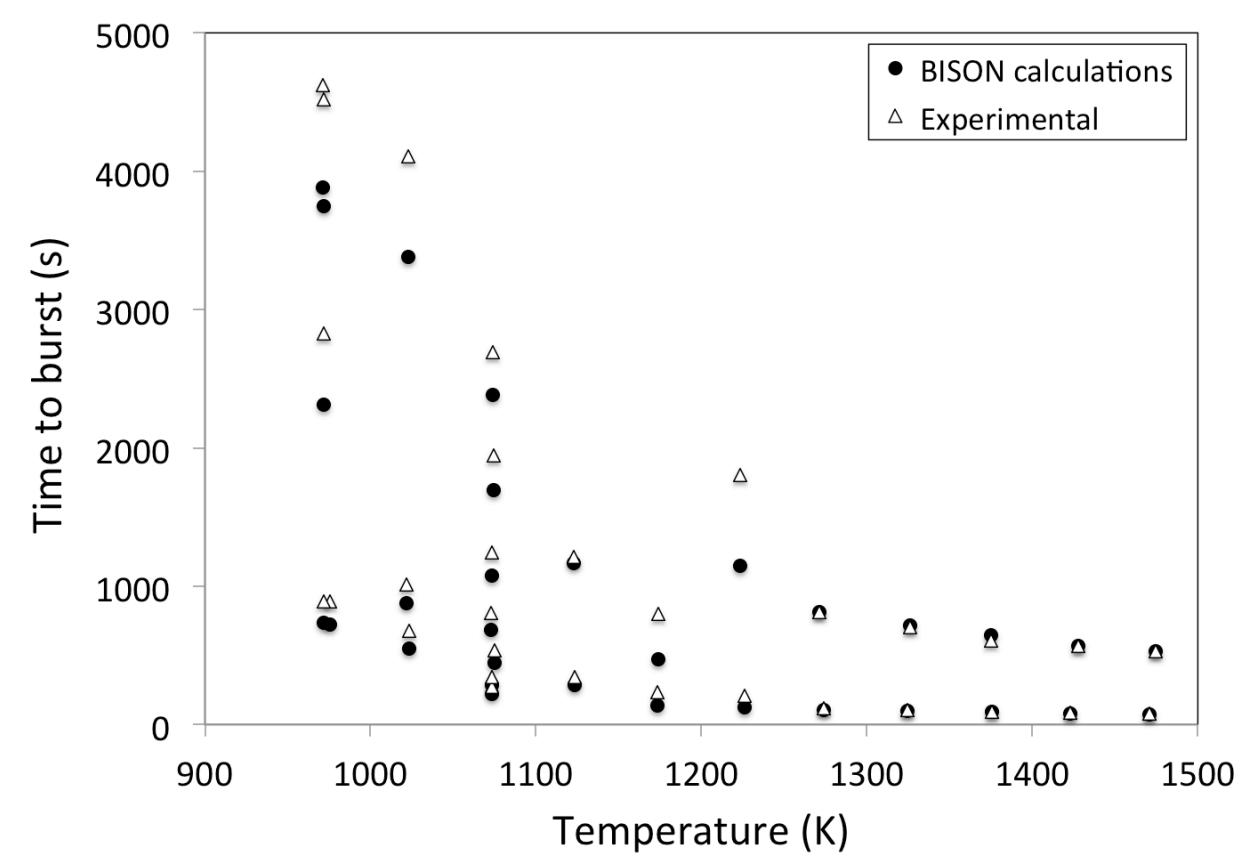

Figure 3.4. Calculated and measured time to burst as a function of test temperature for the PUZRY cases.

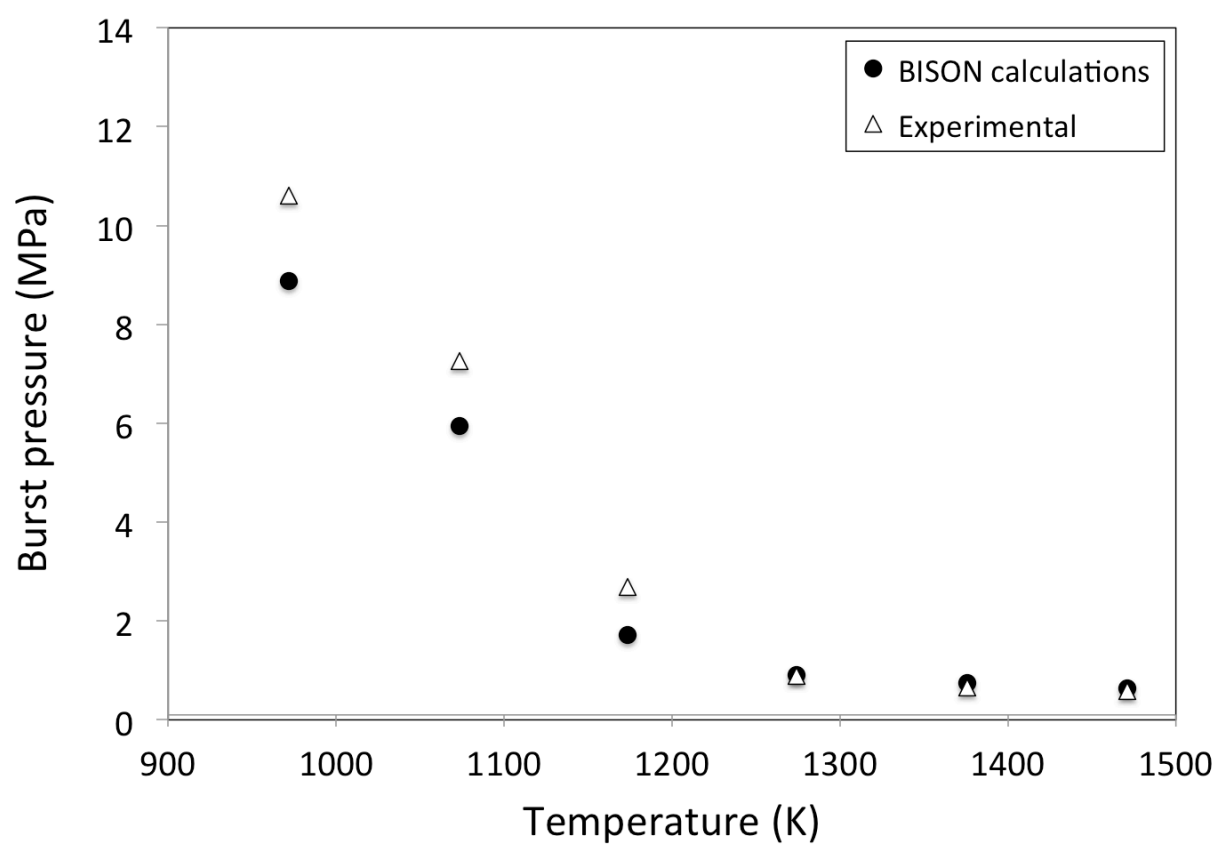

Figure 3.5. Calculated and measured tube inner pressures at burst as a function of test temperature for the 6 PUZRY cases included in FUMAC.

lower temperatures. Higher discrepancies between calculations and experiments at the lower temperatures indicate that deviations may be partly due to anisotropic creep behavior, which is not considered in the BISON model at this time and characterizes alpha- $\mathrm{Zr}$ (i.e., in absence of phase transition to beta-Zr at high temperature, see Section 2.2) $[18,19]$.

Detailed BISON results and experimental data for the 6 PUZRY cases included in FUMAC are tabulated in Table 3.2. 


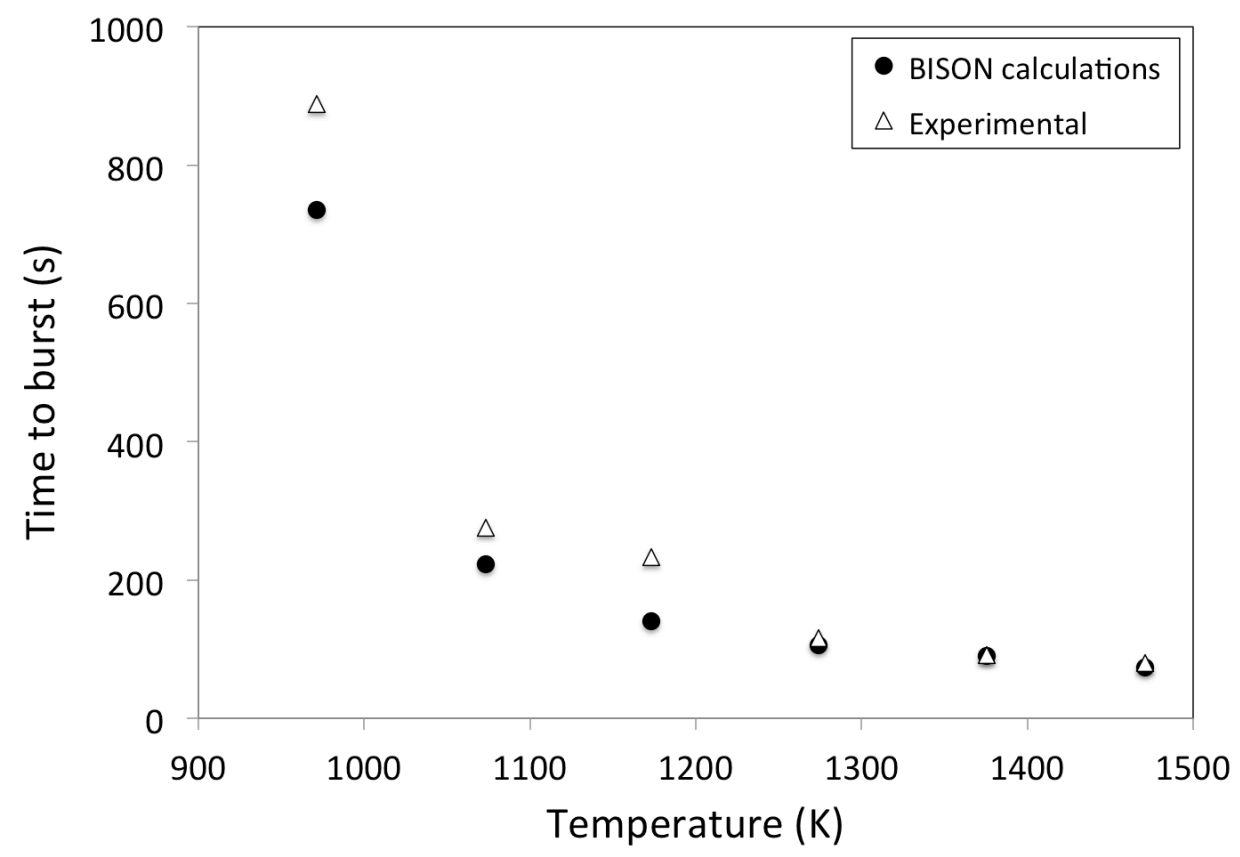

Figure 3.6. Calculated and measured time to burst as a function of test temperature for the 6 PUZRY cases included in FUMAC.

Table 3.2. Experimental data and BISON predictions for the 6 FUMAC cases from the PUZRY experiment. Time to burst is from the beginning of the transient.

\begin{tabular}{cccccccc}
\hline \multirow{2}{*}{ Rod } & Temperature (K) & \multicolumn{2}{c}{ Burst pressure (MPa) } & \multicolumn{2}{c}{ Time to burst (s) } & \multicolumn{2}{c}{ Max hoop strain (\%) } \\
& & Experiment & BISON & Experiment & BISON & Experiment & BISON \\
\hline 8 & 1274 & 0.890 & 0.906 & 116.7 & 105.7 & 80.37 & 80.7 \\
10 & 1376 & 0.653 & 0.737 & 92.0 & 89.8 & 72.76 & 29.9 \\
12 & 1471 & 0.578 & 0.636 & 80.0 & 74.1 & 71.62 & 32.5 \\
18 & 1173 & 2.689 & 1.717 & 233.7 & 140.5 & 74.29 & 42.3 \\
26 & 972 & 10.605 & 8.874 & 888.8 & 735.4 & 100.97 & 81.6 \\
30 & 1074 & 7.251 & 5.951 & 275.7 & 222.5 & 104.28 & 51.3 \\
\hline
\end{tabular}

\subsection{Separate effects cladding tests REBEKA}

The REBEKA separate effects tests [19,20,39] are temperature transient tests in steam performed on single PWR-size Zircaloy-4 tubes at a variety of internal pressures and heating rates. The purpose of the tests was to establish data of cladding ballooning and burst with reference to LOCA conditions.

As mentioned before, although the REBEKA tests were not originally included in FUMAC, BISON simulations are presented here for completeness and including a 3D analysis demonstration, which is of potential interest to the project. 


\section{Description of the tests}

The cladding tubes had a fabricated inner/outer diameter of $9.30 / 10.75 \mathrm{~mm}$, with a $325 \mathrm{~mm}$ heated length, and were heated indirectly by conduction heating from the inside, using an electrically insulated heater rod. A stack of alumina annular pellets $\left(\mathrm{Al}_{2} \mathrm{O}_{3}\right)$ was used to simulate the fuel column. The diametral clearance between the cladding inner diameter and the pellet outer diameter was $0.15 \mathrm{~mm}$. The test parameters covered a range of 1 to $14 \mathrm{MPa}$ for the internal rod (He) pressure and 1 to $30 \mathrm{~K} \mathrm{~s}^{-1}$ for the heating rate. The test atmosphere was almost stagnant steam at atmospheric pressure and at a temperature of $473 \mathrm{~K}$. The cladding temperatures were measured by thermocouples spot-welded on the outer surface of the cladding. More details on the experimental apparatus and conditions are given in $[19,20,39]$.

\section{Setup of the BISON simulations}

The considered cases are modeled considering only the cladding, while the alumina pellets are taken into account by imposing a proper temperature boundary condition at the cladding inner radius, which accounts for the heat transfer through the inner components. For simplicity, only the heated portion of the rods was simulated. The internal electric heating was simulated by a time-dependent Dirichlet temperature boundary condition applied to the tube inner wall and consistent with the experimental conditions. In particular, a parabolic temperature profile symmetric with respect to the tube mid-plane was considered, which results from the uniform axial power generation in the heater rod [39]. To estimate the temperature variation over the heated length of the cladding, simplified calculations of axial heat conduction within the rod and convection to the outer steam atmosphere were performed. Pressure equal to the experimental value was applied at the tube inner wall. A 2-dimensional axisymmetric quadratic (Quad8 elements) mesh was used to model the geometry of the considered rods. In addition, to investigate inherently three-dimensional aspects, such as the effect of azimuthal temperature differences, 3D simulations were conducted employing hexahedral elements (Hex20 elements). Taking advantage of the symmetry of the problem, only the lower half of the heated cladding length was modeled in the $2 \mathrm{D}$ simulations. For the $3 \mathrm{D}$ simulations, a quarter of the cladding circumference was modeled.

The combined overstress and plastic instability criterion for cladding burst failure (Section 2.4) was used for the REBEKA simulations with BISON.

\section{Results for $2 D$ simulations}

Using the 2D axisymmetric model, simulations were conducted of the REBEKA experiments with a heating rate of $1 \mathrm{Ks}^{-1}$, considering the full range of 1 to $14 \mathrm{MPa}$ for the internal cladding pressure. As for the 3D model, only one case is reported here, in order to demonstrate BISON's ability to assess the impact of azimuthal temperature variations on cladding ballooning and burst. 


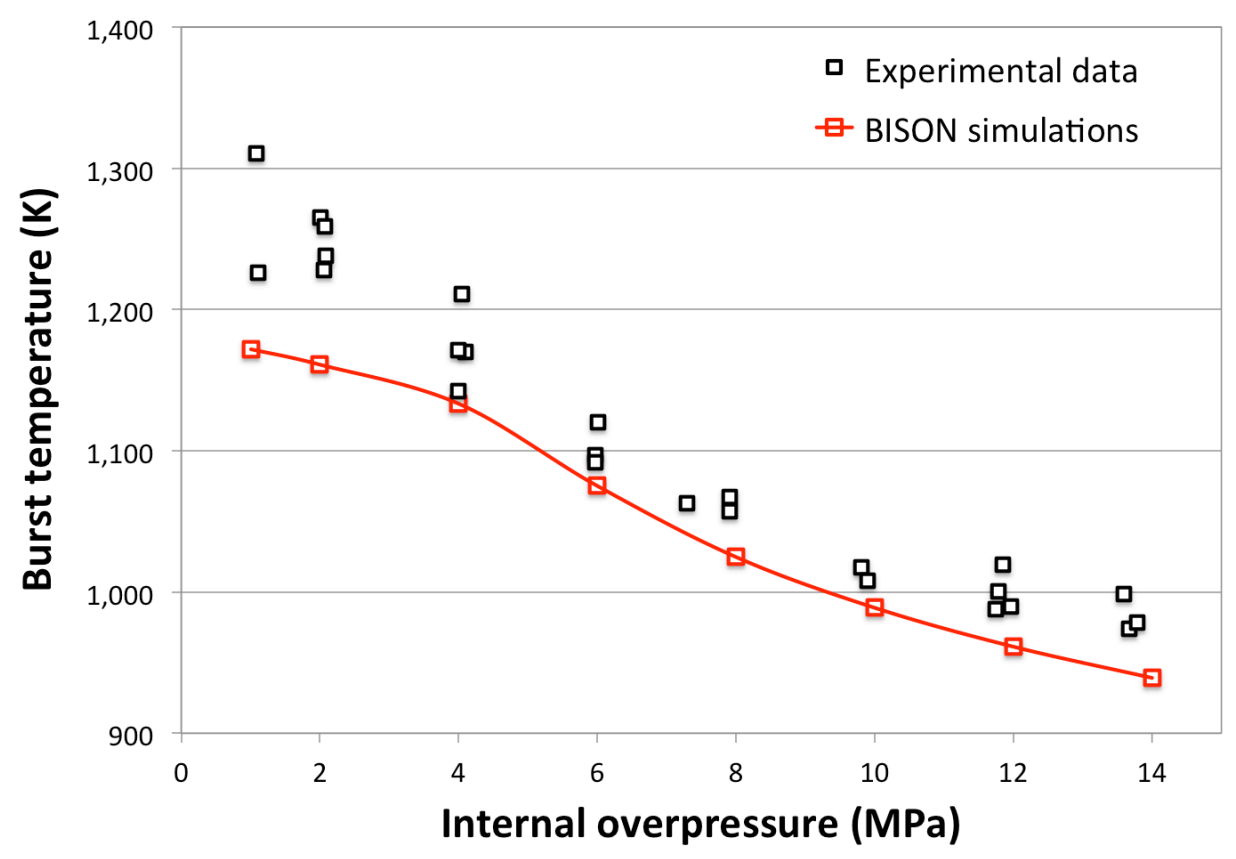

Figure 3.7. Comparison between BISON predictions and experimental data of cladding burst temperature for the simulations of the REBEKA tests with a heating rate of $1 \mathrm{Ks}^{-1}$.

The predictions of burst temperature at the various internal cladding pressures are compared to the available experimental data in Fig. 3.7. The trend of increasing burst temperature with decreasing internal pressure is reproduced, and the quantitative accuracy of predictions is reasonable. Nevertheless, a moderate but systematic under-prediction is observed. Such discrepancies may be due to uncertainties inherent in the cladding mechanics, burst, oxidation and phase transformation modeling, and 3D effects (azimuthal temperature differences) that cannot be captured by $2 \mathrm{D}$ modeling.

\section{Results for $3 D$ simulation}

In addition to the above mentioned boundary conditions applied to the $2 \mathrm{D}$ simulations, in the 3D simulation an azimuthal temperature gradient was applied. A maximum azimuthal temperature variation of $30 \mathrm{~K}$ was considered, in conformity with the experimental indications from thermocouple measurements [20]. The results are presented for the exemplifying case of $10 \mathrm{MPa}$ internal pressure at the time of cladding burst. Figure 3.8 shows contour plots of temperature, creep strain magnitude, and locations where the local stress reached the limiting burst stress. The $3 \mathrm{D}$ simulation reproduces the non-uniform cladding ballooning and a localized burst on the hottest side of the cladding, which is consistent with the experimental observations [20]. Note that the predicted burst temperature is higher (by about $10 \mathrm{~K}$ ) than for the corresponding $2 \mathrm{D}$ simulation, thus indicating that capturing $3 \mathrm{D}$ aspects such as the effect of azimuthal temperature differences is of importance for fuel analysis during LOCA accidents. Further investigation of 3D effects in fuel rod analysis during LOCAs with BISON will be pursued in the future. 

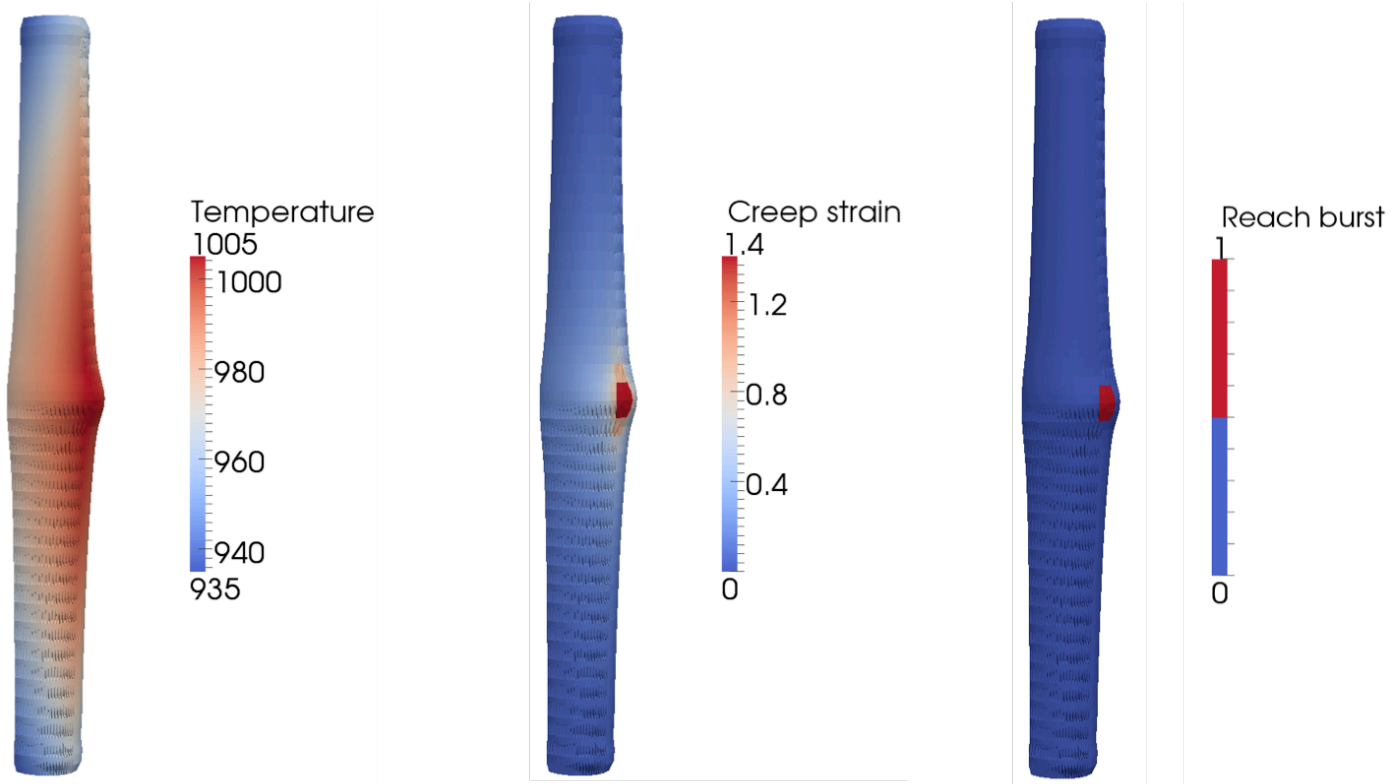

Figure 3.8: Contour plots for the BISON 3D simulation of the REBEKA test with $10 \mathrm{MPa}$ internal pressure at the time of cladding burst. The results for the lower quarter of the heated cladding are mirrored to obtain a full-length, half circumference view. The view is magnified 3 times in the radial direction for improved visualization.

\subsection{QUENCH-L1}

BISON simulations for the QUENCH-L1 experiment [40] were carried out for rods 4 and 7. Experimental data was used as much as possible to develop accurate boundary conditions for the simulations.

\section{Description of the test}

During the QUENCH-L1 test, superheated steam and argon enter a test rod bundle at the bottom of the assembly and flow upward. Quenching water is injected from the bottom of the assembly. The test bundle consists of 21 fuel rod simulators and four corner rods. Each fuel rod simulator is approximately 2.5 meters in length. The heated length of the rod begins at $692.5 \mathrm{~mm}$ from the bottom of the rod, and consists of a central tantalum heater with a diameter of $6 \mathrm{~mm}$ surrounded by $1.575 \mathrm{~mm}$ thick $\mathrm{ZrO}_{2}$ pellets. The plenum separating the heater/pellet combination meant to simulate fuel pellets is filled with krypton gas with a gap between the outer radius of the $\mathrm{ZrO}_{2}$ pellets and the cladding of $0.075 \mathrm{~mm}$. The Zircaloy- 4 clad of $0.725 \mathrm{~mm}$ thickness encases this system. The heated length is $1024 \mathrm{~mm}$ long.

Thermocouples along the surface at axial locations ranging from $-250 \mathrm{~mm}$ to $1250 \mathrm{~mm}$ in increments of $100 \mathrm{~mm}$ as well as those in the plenum of instrumented rods collect temperature data throughout the experiment. Pressure sensors in the plenum as well as the inlet and outlet record internal rod and system pressures respectively.

The experiment began by applying a total power of $3.5 \mathrm{~kW}$ to the electrical bundle. Fuel rod simulators were then individually backfilled to 55 bar and electrical power was rapidly increased to $43 \mathrm{~kW}$ to initiate the transient. This initial power increase was followed by a steady increase to $59 \mathrm{~kW}$ over the next 87 seconds. The power was then rapidly decreased back to $3.5 \mathrm{~kW}$ with water injection at a rate of $100 \mathrm{~g} / \mathrm{s}$ beginning at $207 \mathrm{~s}$. The quench 
progressed toward the top of the coolant channel (bundle bottom at $246 \mathrm{~s}$, ballooned region at $266 \mathrm{~s}$, whole bundle at $293 \mathrm{~s}$ ).

\section{Setup of the BISON simulations}

Rods 4 and 7 were chosen for consideration since they had the largest number of axial thermocouples. Results from the instrumentation were used as applicable to define boundary conditions in the model. The aim of the calculation was to simulate the fuel rod behavior up to burst and compare to measured burst conditions.

A 2D axisymmetric finite element mesh was developed considering only the headed portion of the rods. The Ta heater was included along with the $\mathrm{ZrO}_{2}$ pellets as hollow cylindrical pellets fitting around the heater, and the $\mathrm{Zy}-4$ cladding. Material properties of the $\mathrm{ZrO}_{2}$ pellets were obtained from [40].

The plastic instability criterion for cladding burst failure (Section 2.4) was used for the QUENCH-L1 simulations with BISON.

\section{Development of the boundary conditions}

Pressure and temperature data from the experiment were used to build boundary conditions for BISON. Provided instrument data begins at $-100.6 \mathrm{~s}$ before the initial power ramp begins the transient, but a ramp of temperature and pressure for the system initial conditions is necessary. In addition, the pressure ramp begins well before $-100.6 \mathrm{~s}$ so is digitized directly from the plots in [40] to provide accurate plenum conditions. Boundary conditions on the outside of the cladding depend upon axial position and must be interpolated in a way that both preserves the original data but predicts the data shape accurately between experiment collection points as BISON linearly interpolates between provided data points.

Power provided to the inner and outer rod groups over the course of the experiment is detailed in the collected data and is kept generally the same $(3.5 \mathrm{~kW}$ total $)$ before the transient occurs. A ramp from zero power is included before the pressure ramp to stabilize the system. This ramp, along with the recorded power, is read into the input as a function, and applied as a heat source.

The gas gap pressure is given from -100.6 s through rod bursts and subsequent quenching. The initial rod backfill data are digitized from [40], and obtained values are used as the internal pressure boundary condition from approximately $-3000 \mathrm{~s}$ to $-911 \mathrm{~s}$ at which point the pressure recorded for each rod at $-100.6 \mathrm{~s}$ is held steady. A sudden drop in pressure for each rod at around $50 \mathrm{~s}$ indicates the point at which the cladding fails via the burst mechanism. Informing the simulation from experimental pressure data is problematic around this point, as providing the experiment pressure values to the simulation in cases where the burst time prediction is later than experiment burst time reduces the pressure on the cladding prematurely. To address this, pressures from the beginning of the transient until the burst are fitted to a linear regression model, and this pressure model is used as the plenum pressure boundary condition from $\mathrm{t}=0 \mathrm{~s}$ until predicted burst. Employing this modeling scheme does remove perturbations and the larger the difference between experimental and simulation burst times, the more the model departs from collected data. However, this is viewed as the most viable method to predict extended plenum pressure behavior, as the difference between model and experimental pressure data up until the burst is minimal. By way of example, rod inner pressure experimental data and linear regression model that is used to inform the BISON calculation for rod 4 are shown in Fig. 3.9. 


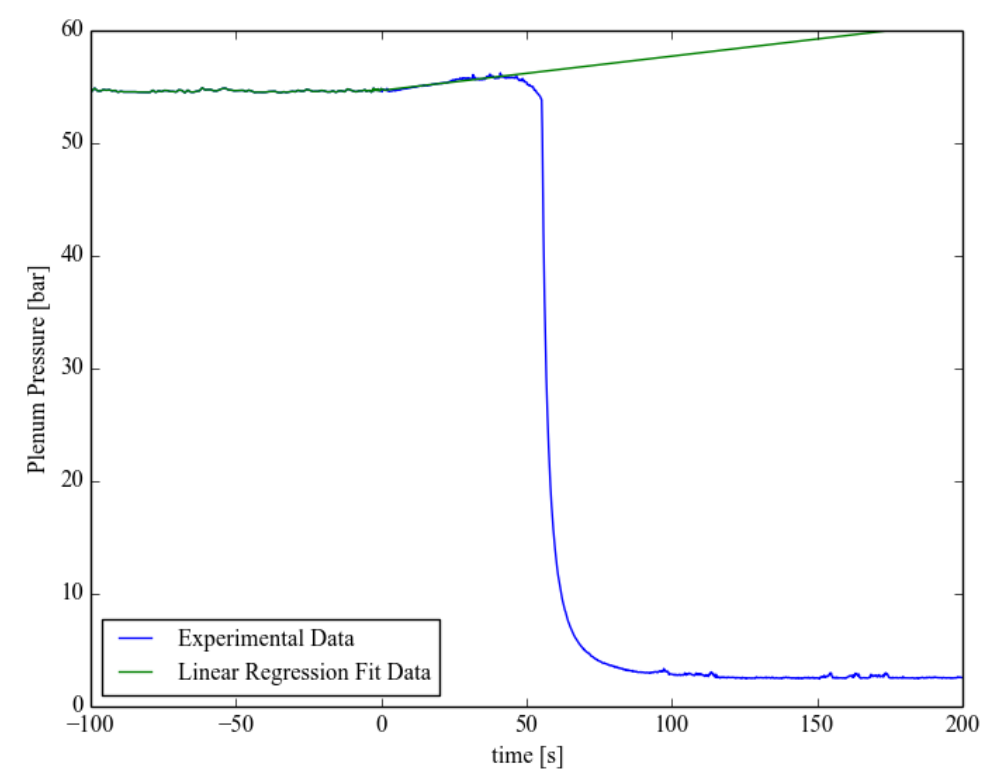

Figure 3.9. Plot of plenum pressure vs. time for Rod 4 .

To build the outer cladding temperature boundary conditions, the sampled cladding surface thermocouple data is conditioned using cubic spline interpolation. Note that the lowest temperature collection point for rod 4 is at $25 \mathrm{~mm}$ while the bottom of the simulated fuel rod begins at $0 \mathrm{~mm}$. To remedy this gap in the data, we assume system conditions are likely to be similar before flowing past the heated length of the fuel rod simulators, and data from one thermocouple recording temperatures from below the heated length of rod 7 is added to the rod 4 data. By way of example, the data and the interpolated values for rod 4 are plotted in Fig. 3.10 at several points during the power ramp.

The final boundary condition the simulation requires is the system pressure along the cladding. Pressures are collected from the inlet and outlet of the cooling loop and values recorded over time are given in [40]. Pressure along the heated length is linearly interpolated as the pressure condition along the heated length.

\section{Results}

Comparison of metrics between the experiment and calculated models are provided in Tables 3.3 and 3.4. Cladding burst is predicted to occur at 55.8 and $68.4 \mathrm{~s}$ after beginning the transient heating phase for rods 4 and 7 , respectively. The experimental failures were observed at 55.2 and $54.4 \mathrm{~s}$ for rods 4 and 7, respectively. The hoop strain is under-predicted in both cases by about a factor of 3 . Temperature and pressure calculations are very close to the measured values at the time of failure. Both predicted burst elevations are higher than the experimental locations but are consistent with the experiment in that burst elevation occurs at the upper end of the fuel rod simulators. 

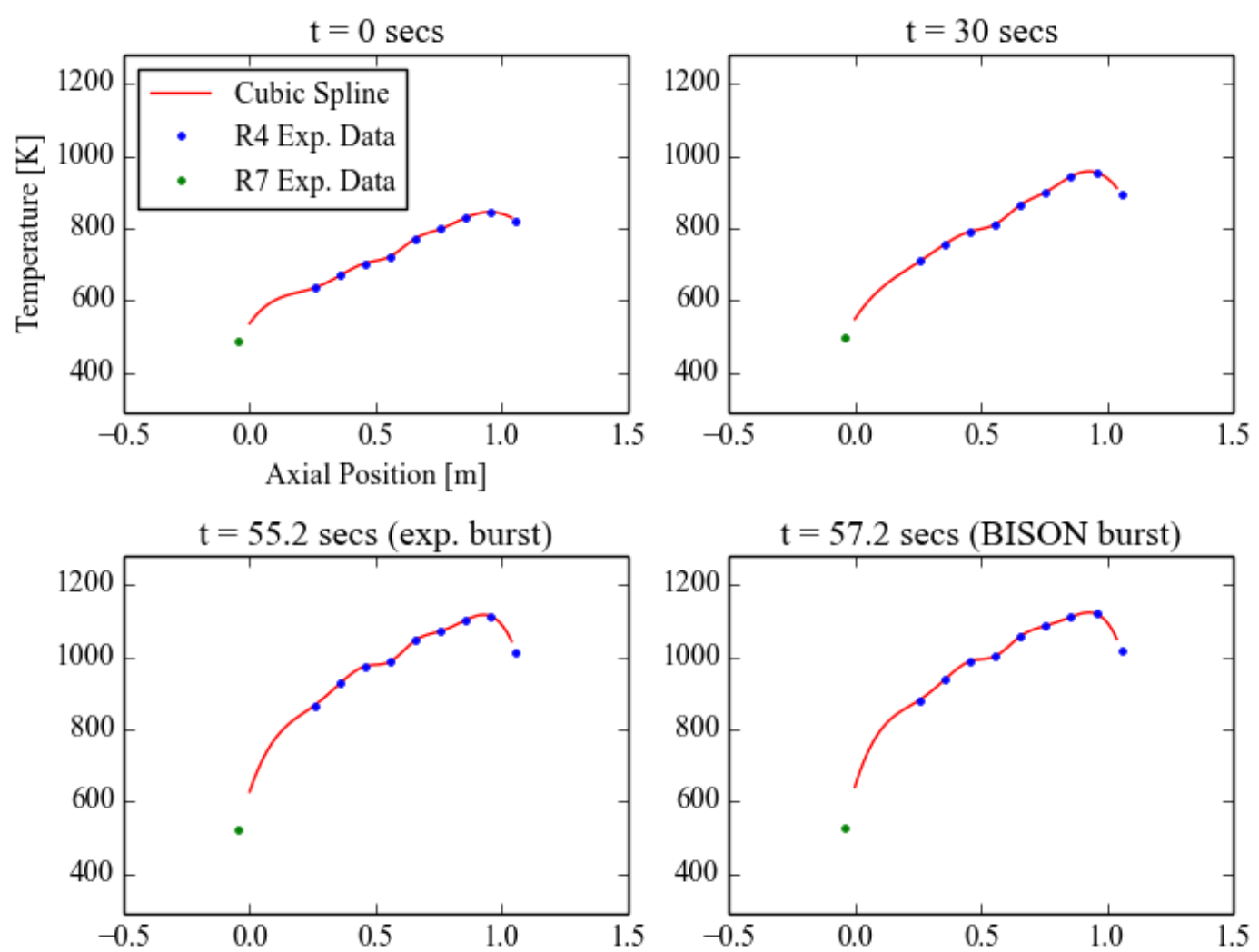

Figure 3.10: Rod 4 Temperature vs. axial location on outside of clad.

Table 3.3. Experimental and simulation results for QUENCH-L1 rod 4

\begin{tabular}{lll}
\hline & Experiment & BISON \\
\hline Burst time (s) & 55.2 & 55.8 \\
Burst elevation (mm) & 979 & 927 \\
Burst inner pressure (bar) & 53.9 & 56.4 \\
Temperature at 950 mm (K) & 1154 & 1117 \\
Max hoop strain (\%) & 28.9 & 8.9 \\
Max rod diameter (mm) & 15 & 11.6 \\
Min rod diameter (mm) & 13 & 10.8 \\
\hline
\end{tabular}

Table 3.4. Experimental and simulation results for QUENCH-L1 rod 7

\begin{tabular}{lll}
\hline & Experiment & BISON \\
\hline Burst time (s) & 54.4 & 68.4 \\
Burst elevation (mm) & 953 & 800 \\
Burst inner pressure (bar) & 55.1 & 56.6 \\
Temperature at 950 mm (K) & 1074 & 1077 \\
Max hoop strain (\%) & 24.8 & 8.1 \\
Max rod diameter (mm) & 14.7 & 11.5 \\
Min rod diameter (mm) & 12.5 & 10.8 \\
\hline
\end{tabular}




\subsection{Halden IFA-650.2}

LOCA tests at Halden (IFA-650 series) are integral in-pile single rod tests. Relative to separate effects tests, they also provide information on the integral behavior of a fuel rod during a LOCA accident. The second trial test IFA-650.2 [41] was modeled with BISON.

\section{Description of the test}

The test was carried out using a fresh, pressurized PWR rod and low fission power to achieve the desired temperature conditions. The rod plenum volume was made relatively large to be able to maintain stable pressure conditions during ballooning. The fabrication characteristics of the IFA-650.2 fuel rod are reported in Table 3.5.

The fuel rod was located in a standard high-pressure flask in the IFA-650 test rig in the Halden reactor. A heater surrounding the rod was used to simulate the heat from adjacent rods. The flask was connected to a high-pressure heavy water loop and a blowdown system. During normal operation prior to the LOCA test, the rig was connected to the loop and forced circulation flow conditions existed. Then, the rig was disconnected. A natural convection phase began, with water flowing up between the fuel rod and flow separator (with heater) and down between flow separator and flask wall. Full pressure still existed in the rig. LOCA was initiated by opening the valves leading to the blowdown tank (blowdown phase). The initial pressure in the loop was $\sim 7 \mathrm{MPa}$ and the counterpressure in the blowdown tank was $\sim 0.2$ $\mathrm{MPa}$. The channel pressure decreased to 3-4 bars, and the rig was practically emptied of water within 30-40 seconds. Stagnant superheated steam surrounding the rod provided inadequate cooling and the cladding temperature increased quickly (heat-up phase). A low fission power of $2.3 \mathrm{~kW} / \mathrm{m}$ was used to simulate decay heat and achieve the desired temperature conditions. Cladding ballooning and burst rupture occurred during the heat up phase. Measured cladding temperature at burst was $\sim 815 \mathrm{C}$. The test was ended by a reactor scram.

Table 3.5. Design data of IFA-650.2 fuel rod [41].

\begin{tabular}{lll}
\hline Fuel material & & $\mathrm{UO}_{2}$ \\
Fuel density & $\% \mathrm{TD}$ & 95.0 \\
${ }^{235} \mathrm{U}$ enrichment & $\mathrm{wt} \%$ & 2.0 \\
Active stack length & $\mathrm{mm}$ & 500 \\
Pellet OD & $\mathrm{mm}$ & 8.29 \\
Pellet ID & $\mathrm{mm}$ & 0 \\
\hline Cladding material & & $\mathrm{Zy}-4$ \\
Cladding ID & $\mathrm{mm}$ & 8.36 \\
Cladding OD & $\mathrm{mm}$ & 9.50 \\
Diametral gap & $\mu \mathrm{m}$ & 70 \\
Free volume & $\mathrm{cm}$ & 17.4 \\
\hline Fill gas & & $\mathrm{He}$ \\
Fill gas pressure & $\mathrm{MPa}$ & 4.0 \\
\hline
\end{tabular}




\section{Setup of the BISON simulation}

The rod geometry was modeled following the design specifications from [41] (Table 3.5). The enriched fuel pellet column was represented with a smeared fuel column. Natural $\mathrm{UO}_{2}$ pellets at the top and bottom of the fuel stack were also included. A single rod upper plenum was considered, whose volume is the sum of the various plenum volumes in the more complex real geometry [41]. A 2D axisymmetric quadratic finite element mesh was used.

The combined overstress and plastic instability criterion for cladding burst failure (Section 2.4) was adopted for the IFA-650.2 simulation.

The boundary conditions (BCs) in terms of linear heat rate and rig pressure were derived from the raw data from provided by the Halden Project and tabulated for usage in BISON. Temperature BCs at the cladding outer surface were evaluated based on cladding outer temperatures, which were measured at two axial positions during the experiment. In particular, axial temperature profiles at the clad outer surface were obtained using some simplifying assumptions and imposing coincidence with the measured temperatures at measurement axial locations. The obtained profiles were used as outer cladding temperature BCs in absence of detailed thermal-hydraulics calculations. The procedure and assumptions adopted for the calculation of the temperature BCs used for the BISON simulations of IFA650.2 at INL was first discussed on the FUMAC website in advance of the RCM2 [42]. A detailed description is given hereinafter.

\section{Determination of the thermal boundary conditions}

Clad outer temperature axial profiles are obtained based on the following assumptions:

- The effect of radiation is lumped into an "effective" heat transfer coefficient. This simplification is based on linearizing the radiative heat transfer law $q^{\prime \prime} \propto\left(T_{1}^{4}-T_{2}^{4}\right)$ to $q^{\prime \prime} \propto h_{\text {rad }}\left(T_{1}-T_{2}\right)$. Here $q^{\prime \prime}$ is the heat flux and $h_{\text {rad }} \propto T_{\text {average }}^{3}$ and has units of a heat transfer coefficient. The linearized equation is accurate if $T_{1}$ and $T_{2}$ are close enough. Under this assumption, the axial clad temperature profile can be written in a form as if heat transfer were purely convective

$T(z)=T_{\text {cool }}(z)+q^{\prime \prime}(z) / h_{e f f}(z)$

where $z$ is the axial coordinate, $T_{\text {cool }}$ the coolant temperature, and $h_{\text {eff }}$ is the "effective" heat transfer coefficient (convection + radiation).

- The coolant temperature is approximated as the heater temperature at the axial position $z$. This is estimated based on the Halden data of measured heater temperature at two axial locations and a linear interpolation.

- The heat flux is proportional to the local linear heat rate, i.e., $q^{\prime \prime}(z) \propto q^{\prime}(z)$. This is reasonable provided that the coolant channel conditions are reasonably uniform along the rod and that no significant axial fuel relocation takes place during the test. From this assumption and Eq. 3.1, it follows:

$T(z)=T_{\text {cool }}(z)+q^{\prime}(z) / h^{*}(z)$

where $h^{*}(z)=k h_{e f f}(z)$ and $k$ is a constant. The local linear heat rate, $q^{\prime}(z)$, is obtained from the Halden data. 
- $h^{*}(z)$ is determined based on the measured cladding temperatures. For this purpose, $h^{*}$ is assumed to vary linearly along $z$

$h^{*}(z)=A z+B$

- The two equations needed to determine the coefficients $A$ and $B$ are the conditions of $T(z)$ (Eq. 3.2) being equal to the measured temperatures at the two measurement locations (thermocouples at clad outer wall).

The strongest simplification in the above approach is taking a linear fit of the heat transfer coefficient along the axial direction. When radiation is dominant, $h^{*}(z) \propto T_{\text {average }}^{3}$, approximately, which likely has a maximum at peak power position. However, the above approach based on measured temperatures may be accurate enough in view of the uncertainties involved in determining thermal-hydraulic boundary conditions.

Figures 3.11 to 3.14 show the estimated clad outer temperature profiles at selected instants during different phases of the experiment. Using the measured temperatures and an axially varying heat transfer coefficient, actually, allows one to capture effects such as the higher temperature in the lower part of the rod during the blowdown phase, which are difficult to explain [41] or reproduce through thermal-hydraulics calculations.

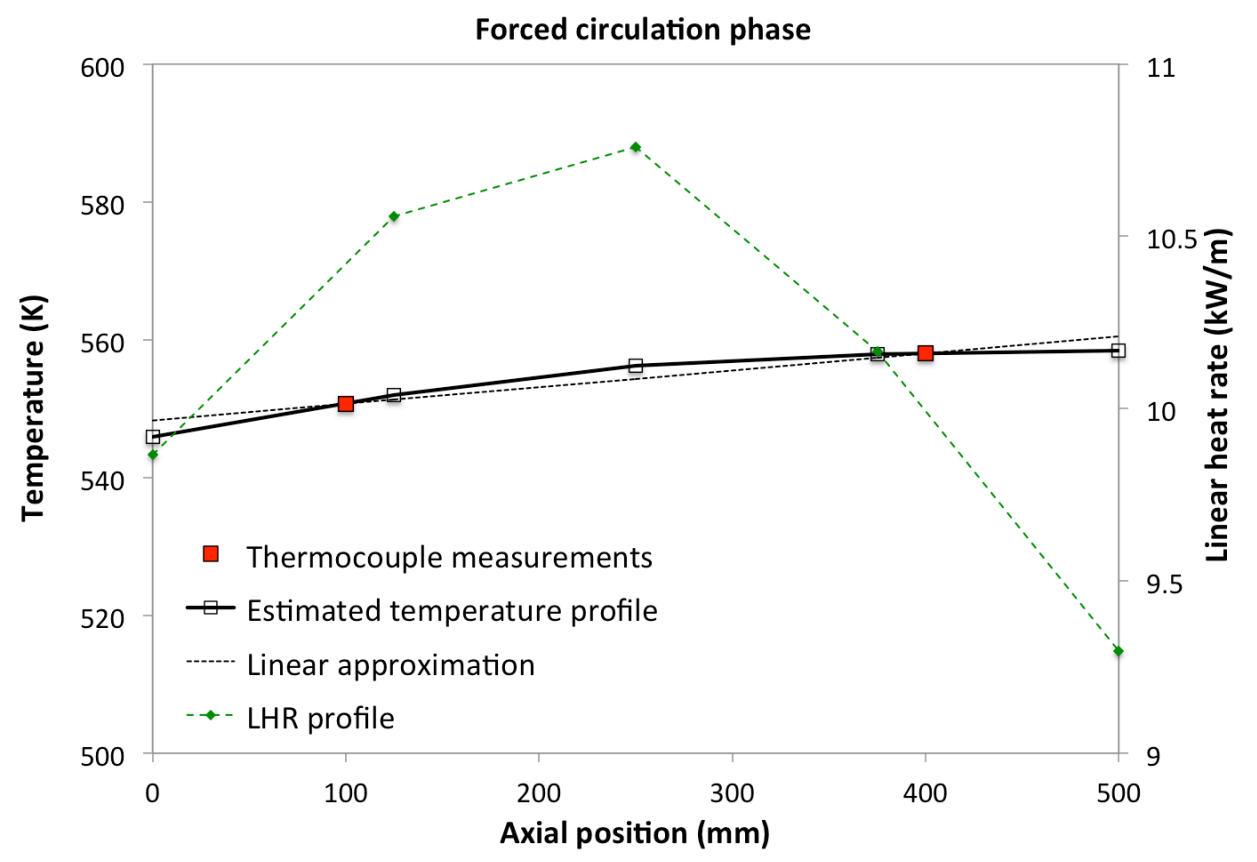

Figure 3.11. Estimated axial temperature profile at cladding outer surface at an instant during the forced circulation phase of the IFA-650.2 experiment. The measured temperatures at the thermocouple locations and the linear heat rate (LHR) profile interpolated from Halden data are also shown. 


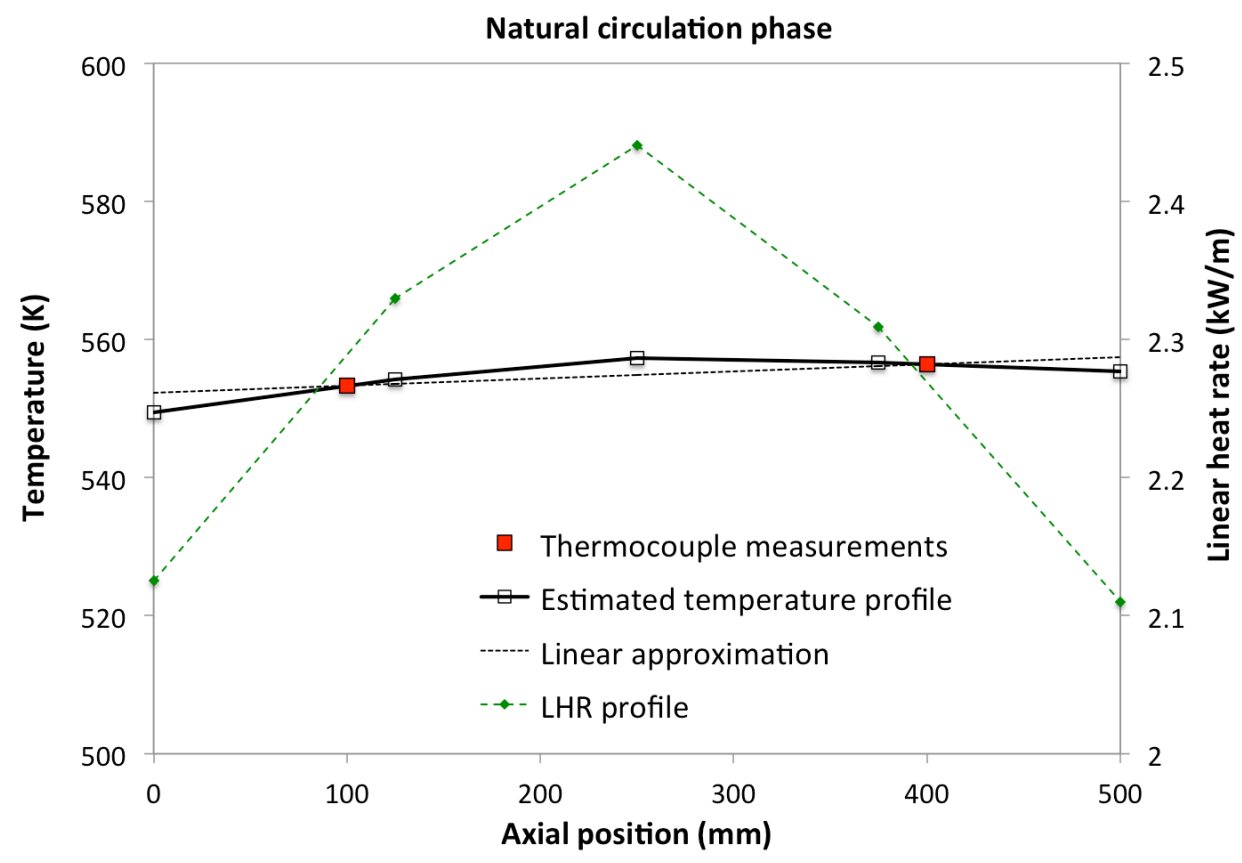

Figure 3.12. Estimated axial temperature profile at cladding outer surface at an instant during the natural circulation phase of the IFA-650.2 experiment. The measured temperatures at the thermocouple locations and the linear heat rate (LHR) profile interpolated from Halden data are also shown.

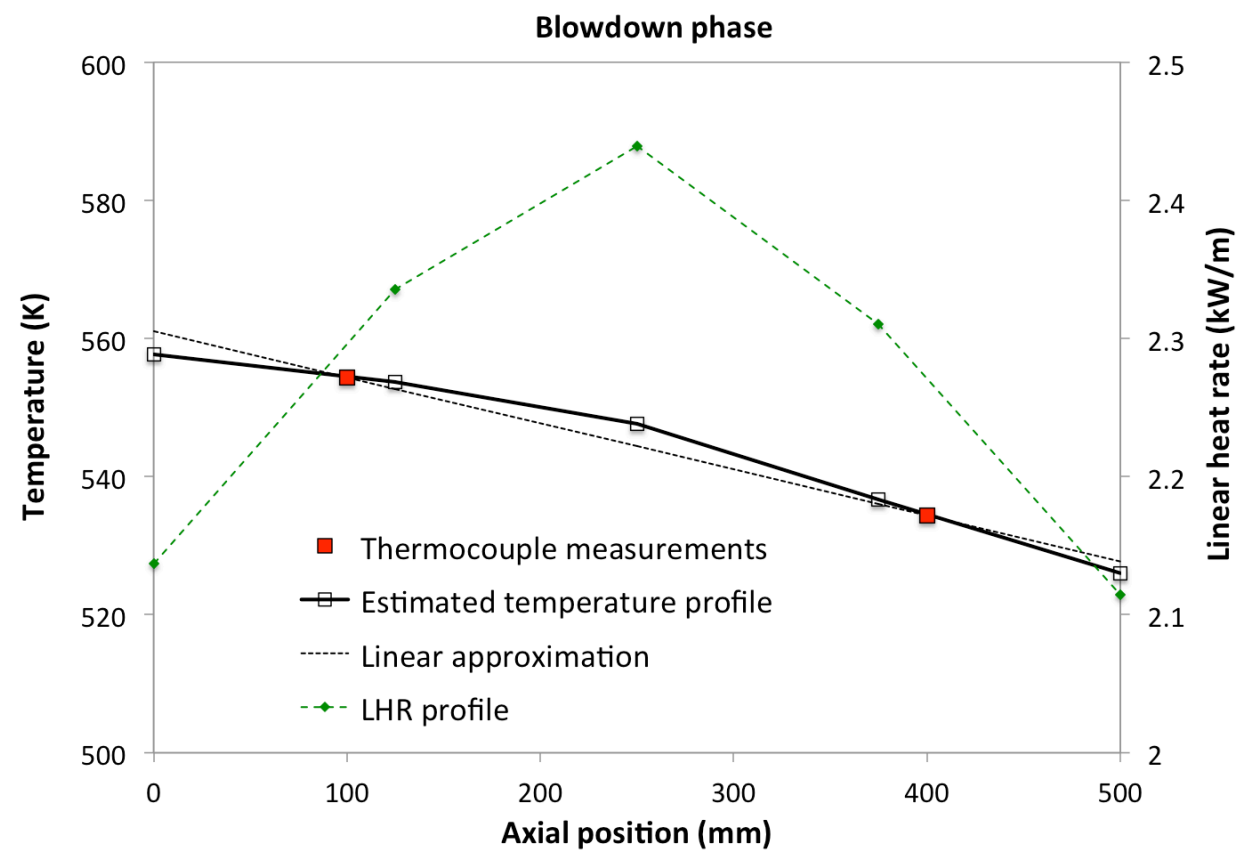

Figure 3.13. Estimated axial temperature profile at cladding outer surface at an instant during the blowdown phase of the IFA-650.2 experiment. The measured temperatures at the thermocouple locations and the linear heat rate (LHR) profile interpolated from Halden data are also shown. 


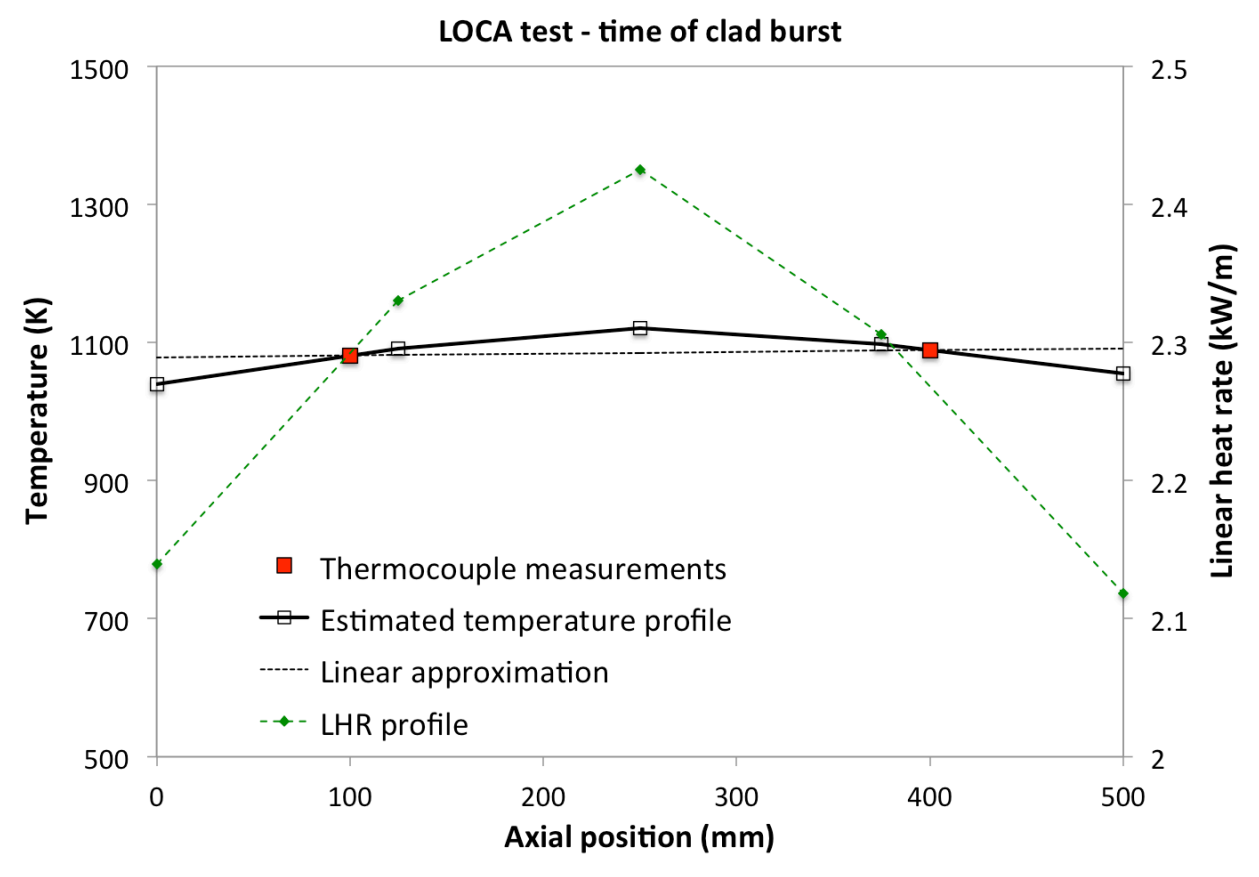

Figure 3.14. Estimated axial temperature profile at cladding outer surface at the time of cladding burst for the IFA-650.2 experiment. The measured temperatures at the thermocouple locations and the linear heat rate (LHR) profile interpolated from Halden data are also shown.

\section{Results}

In Figure 3.15, calculated inner pin pressure during the LOCA transient is compared to the on-line experimental measurement, with predicted and experimental time to burst being also illustrated. The comparison points out that both quantities are predicted reasonably well by BISON. Rod pressure is moderately over-predicted during the heat-up phase of the test, which may be ascribed to discrepancies in the calculated plenum temperature and/or evolution of fuel rod inner volume during ballooning.

In this work, cladding temperature boundary conditions are determined based off the measured cladding outer temperatures, as detailed above. Clearly, temperature BCs at the cladding also affect plenum temperature and in turn, plenum pressure. The small "dip" in the calculated pressure shown in Fig. 3.15 at a time of around $30 \mathrm{~s}$ is due to a corresponding dip in the measured cladding outer temperatures (in particular, from the thermocouples at the upper axial position) from the Halden data. This behavior will require further investigation.

Fission gas release is very low due to the test being performed with a fresh fuel rod and is not expected to affect rod pressure significantly.

Calculated time to burst is $\sim 91.1 \mathrm{~s}$ after blowdown, i.e., about $7 \mathrm{~s}$ before the experimental time to burst ( $\sim 98.5 \mathrm{~s}$ after blowdown). 


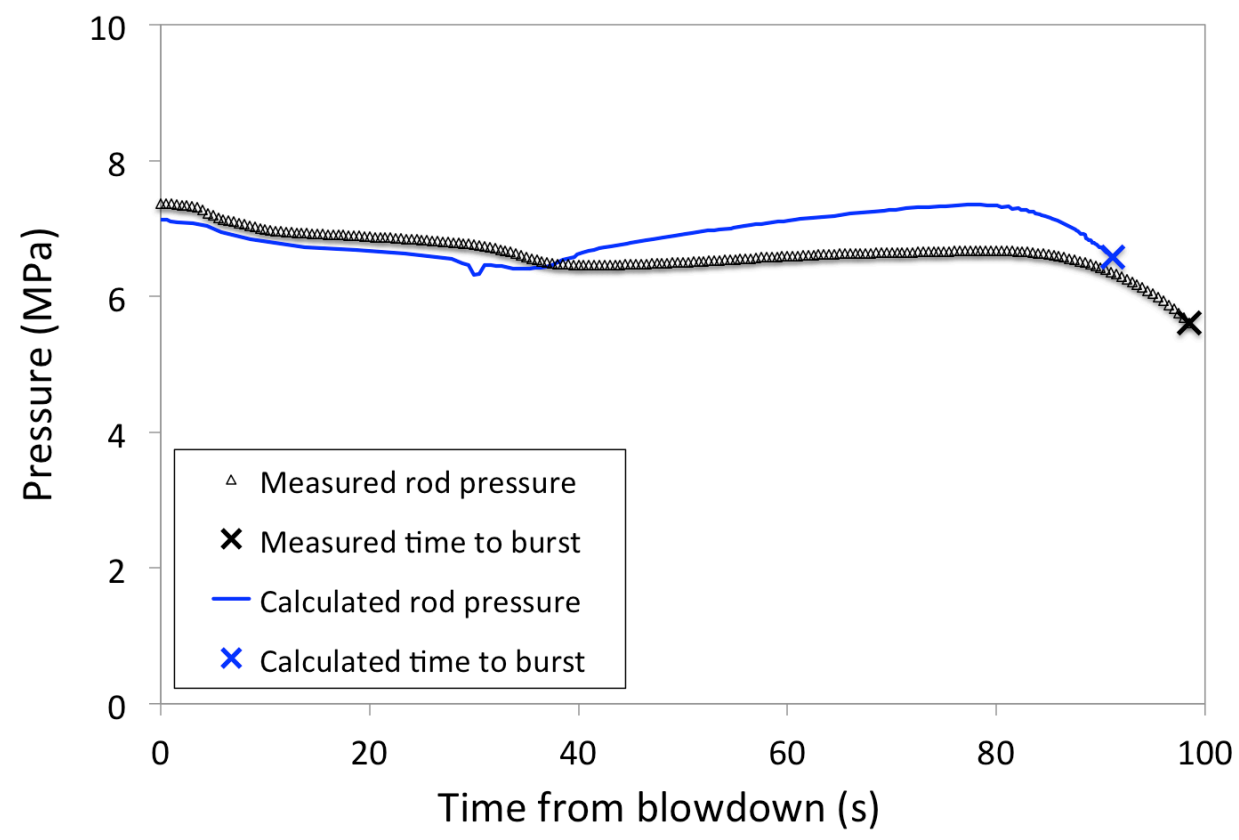

Figure 3.15. Comparison between measured and calculated fuel rod inner pressure and time to cladding bust for the Halden IFA-650.2 test. Time zero corresponds to the beginning of the blowdown phase.

\subsection{Halden IFA-650.10}

The FUMAC priority case Halden IFA-650.10 [43] has been simulated with BISON. The developed BISON computational model included a $2 \mathrm{D}$ geometric representation of the IFA$650.10 \mathrm{rod}$, consistent with the design specifications provided by the Halden Project, power histories and coolant conditions from the beginning of life through the commercial base irradiation and the LOCA test. Furthermore, the simulation was informed with the thermal boundary conditions calculated with the SOCRAT code and provided through FUMAC [44]. We exercised this computational model to obtain a BISON simulation of IFA-650.10 through all the phases of the experiment and up to cladding burst failure.

\section{Description of the test}

The tenth Halden LOCA test, i.e., IFA-650.10 [43] was carried out using a segment of a PWR rod that had been irradiated in a commercial PWR (Gravelines 5, $900 \mathrm{MWe}$, EDF, France) up to a burn-up of $61 \mathrm{MWd} / \mathrm{kgU}$. During the test a low fission power $(25 \mathrm{~W} / \mathrm{cm})$ was used to achieve the desired conditions for high cladding temperatures, ballooning and oxidation. A heater surrounding the rod and operating at $12 \mathrm{~W} / \mathrm{cm}$ was used for simulating the heat from adjacent rods. The average cladding temperature increase rate during the heat-up was around $8 \mathrm{~K} / \mathrm{s}$. Cladding failure occurred $\sim 249$ seconds after blowdown at a cladding temperature of $\sim 1025 \mathrm{~K}$. The fabrication characteristics of the IFA-650.10 fuel rod are reported in Table 3.6.

The fuel rod was located in a high-pressure flask in the IFA-650 test rig, which was connected to a high-pressure heavy water loop and a blowdown system. Cladding temperature is influenced by both rod and heater powers. The flask was surrounded by a shroud and was placed inside the Halden reactor. The annulus between the shroud and the flask is filled with moderator (heavy water) at a pressure of 34 bar and a temperature of $235 \mathrm{C}$. One cladding 
Table 3.6. Design data of the IFA-650.10 fuel rod [43,45].

\begin{tabular}{lll}
\hline Fuel material & & $\mathrm{UO}_{2}$ \\
Fuel density & $\% \mathrm{TD}$ & 95.32 \\
${ }^{235} \mathrm{U}$ enrichment & $\mathrm{wt} \%$ & 4.487 \\
Active stack length & $\mathrm{mm}$ & 440 \\
Pellet OD & $\mathrm{mm}$ & $8.21^{*}$ \\
Pellet ID & $\mathrm{mm}$ & 0 \\
Pellet length & $\mathrm{mm}$ & 10 \\
\hline Cladding material & & $\mathrm{Zy}-4$ \\
Cladding ID & $\mathrm{mm}$ & 8.36 \\
Cladding OD & $\mathrm{mm}$ & 9.50 \\
Diametral gap & $\mu \mathrm{m}$ & 150 \\
Free volume & $\mathrm{cm}{ }^{3}$ & 17 \\
\hline Fill gas & \multicolumn{3}{c}{$95 \% \mathrm{Ar}, 5 \% \mathrm{He}$} \\
Fill gas pressure & $\mathrm{MPa}$ & 4.0 \\
* For consistency with the fuel-cladding gap width $[43,45]$.
\end{tabular}

surface thermocouple was located $9.5 \mathrm{~cm}$ above the fuel stack bottom, and the other two were attached $8 \mathrm{~cm}$ below the top of the stack. The rig instrumentation also included a fuel pressure sensor and thermocouples at the inlet and outlet of the rig to measure the coolant temperatures.

The experimental procedure for the IFA-650.10 test is detailed below [43]. Note that we refer here to the LOCA test performed in the Halden reactor on the pre-irradiated, refabricated PWR fuel rod. In the BISON simulation, we also considered the commercial base irradiation preceding the test.

The general test scheme of IFA-650.10 consisted of the following phases:

- Preparatory phases. The test started with a preparatory irradiation with effective water cooling, consisting of a forced circulation phase followed by a natural circulation phase. The forced circulation phase started with steady state operation at a linear heat generation rate (LHGR) of $120-130 \mathrm{~W} / \mathrm{cm}$, with the outer loop connected and the pressure in the loop set to $\sim 70$ bar. Then the LHGR was decreased to $\sim 25 \mathrm{~W} / \mathrm{cm}$. The power levels were chosen based on the previous test runs and pre-calculations to achieve the target peak cladding temperature (PCT) of $850 \mathrm{C}$ during the heat-up phase of the test. Then the flow regime was switched to natural circulation by disconnecting the rig from the outer loop. Full pressure still existed in the rig. Temperatures in the rig were left to stabilize for three minutes before blowdown.

- Blowdown phase. Valves to the dump tank were opened (blowdown). The channel pressure decreased rapidly to $\sim 4$ bar as water flew out of the pressure flask. The rig was practically emptied of water in $\sim 71 \mathrm{~s}$, which corresponds to the end of the blowdown phase (beginning of the dry phase).

- Dry or heat-up phase. Stagnant superheated steam surrounding the test rod provided inadequate cooling and the fuel cladding temperature increased quickly. Much of the heat removal from the test rod is by radiation to the surrounding heater. Ballooning and burst occurred during the heat-up phase and were detected from pressure and 
temperature signals (burst at $\sim 1025 \mathrm{~K}, \sim 249 \mathrm{~s}$ after blowdown). The test was ended by a reactor scram $418 \mathrm{~s}$ after blowdown.

\section{The BISON computational model for IFA-650.10}

A 2D BISON model of the IFA-650.2 fuel rod was constructed. The fuel pellet stack was meshed as a smeared column. The plenum length was adjusted such that the initial rod inner volume was consistent with the rod data $[43,45]$. For simplicity, the base irradiation was simulated on the geometry of the refabricated IFA-650.10 rod rather than on the geometry of the original commercial mother rod. Refabrication in BISON was accounted for by specifying the temperature, pressure, and volume of the rod filling gas at refabrication.

The plastic instability criterion for cladding burst failure (Section 2.4) was chosen for this simulation, as it was found to be the most appropriate for the analysis of pre-irradiated fuel rod experiments.

\section{Time-dependent boundary conditions}

Simulation of the commercial base irradiation was included in the BISON analysis of IFA650.10. The power history for the base irradiation was digitized from the chart provided within FUMAC [46] and tabulated for usage as input to BISON. For the coolant conditions during the base irradiation, typical PWR parameters were adopted, i.e., water at a pressure of 15.5 $\mathrm{MPa}$, an inlet temperature of $580 \mathrm{~K}$ and an inlet mass flux of $3800 \mathrm{~kg} / \mathrm{m}^{2}-\mathrm{s}$ was considered. The heat transfer from the cladding to the coolant was modeled using BISON's internal coolant channel model for convective heat transfer under PWR conditions.

The Halden test began with preparatory phases of fuel rod irradiation under coolant conditions of forced circulation, first, and natural circulation, afterwards. Since the SOCRAT calculations do not cover these preparatory phases fully [44], to determine the thermal boundary conditions we adopted a pragmatic approach in which we used the measured temperatures available from the Halden data. In particular, for these initial phases of the experiment, we considered an axially flat temperature profile, with the (time dependent) temperature value being the average of the temperature data measured at two different axial locations. The temperature profile along the plenum length is also considered as flat, with the temperature value being equal to the temperature measured by the plenum thermocouple. This approach guarantees good accuracy as the temperature values are derived directly from the measurements. The downside of this approach is that axial temperature peaking (which is associated with power peaking) is not allowed as an axially flat profile is used. This makes such an approach less suitable for the post-blowdown phases of the test, when cladding ballooning occurs that presents an axial dependence (localized ballooning and burst in correspondence of the hottest axial position). This is a consequence of the axial temperature peaking in the cladding and the strong temperature dependence of Zircaloy thermal creep and the associated cladding ballooning. Hence, more detailed thermal boundary conditions are needed for the post-blowdown phases.

The SOCRAT calculated cladding outer temperatures were used from a time $280 \mathrm{~s}$ before the beginning of blowdown onwards (i.e., for the time period where SOCRAT calculated temperatures are available [44]). Figure 3.16 shows the comparison of the cladding outer temperatures compared to the Halden measurements at the axial locations where the measurements were performed. Temperatures are the same as shown in [44] and confirm that the SOCRAT data were supplied correctly to BISON. 
As for the linear heat generation rate (LHGR) history for the rod, this was obtained from the Halden raw data and tabulated for usage in BISON. As data are provided at different axial locations, axial linear interpolation was used.

Note that the experimental transient continued beyond the time of burst, but we stop the simulation at burst time. After burst, factors such as the geometry of the burst opening, fuel rod depressurization, and possible fuel dispersal all affect fuel rod behavior, and are not accounted for in BISON. In this work, we rather focus on predicting pre-burst fuel rod behavior (temperatures, ballooning) as well as the time to burst.

Results

Figure 3.17 shows contour plots of calculated fuel temperatures in the fuel rod at the time of predicted cladding burst failure. Besides the full rod, separate plots for the fuel and cladding are shown with specific color scales. The cladding reaches very high temperatures compared to normal PWR operation values of around $600 \mathrm{~K}$ because of the degraded heat transfer to the coolant during a LOCA that ultimately causes cladding heat-up and ballooning due to thermal creep.

Figure 3.18 shows a contour plot of calculated hoop strain at the time of predicted burst. The figure demonstrates how cladding ballooning, with large cladding strain and a maximum localized near the axial mid-plane of the fuel stack, is reproduced by BISON.

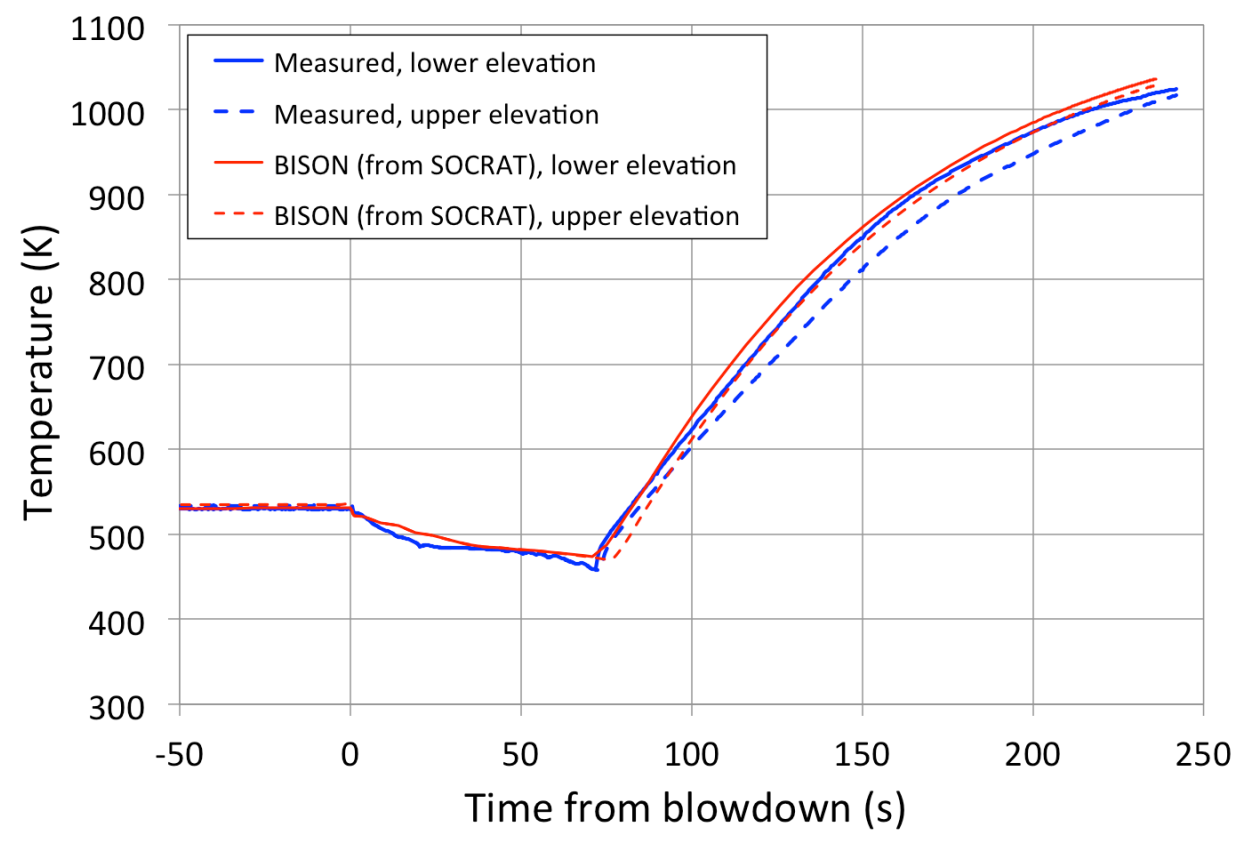

Figure 3.16 Measured cladding outer temperatures at two different axial locations in the IFA650.10 rod during the post-blowdown phases of the test and corresponding temperatures in BISON (prescribed, from the SOCRAT calculations [44]) 


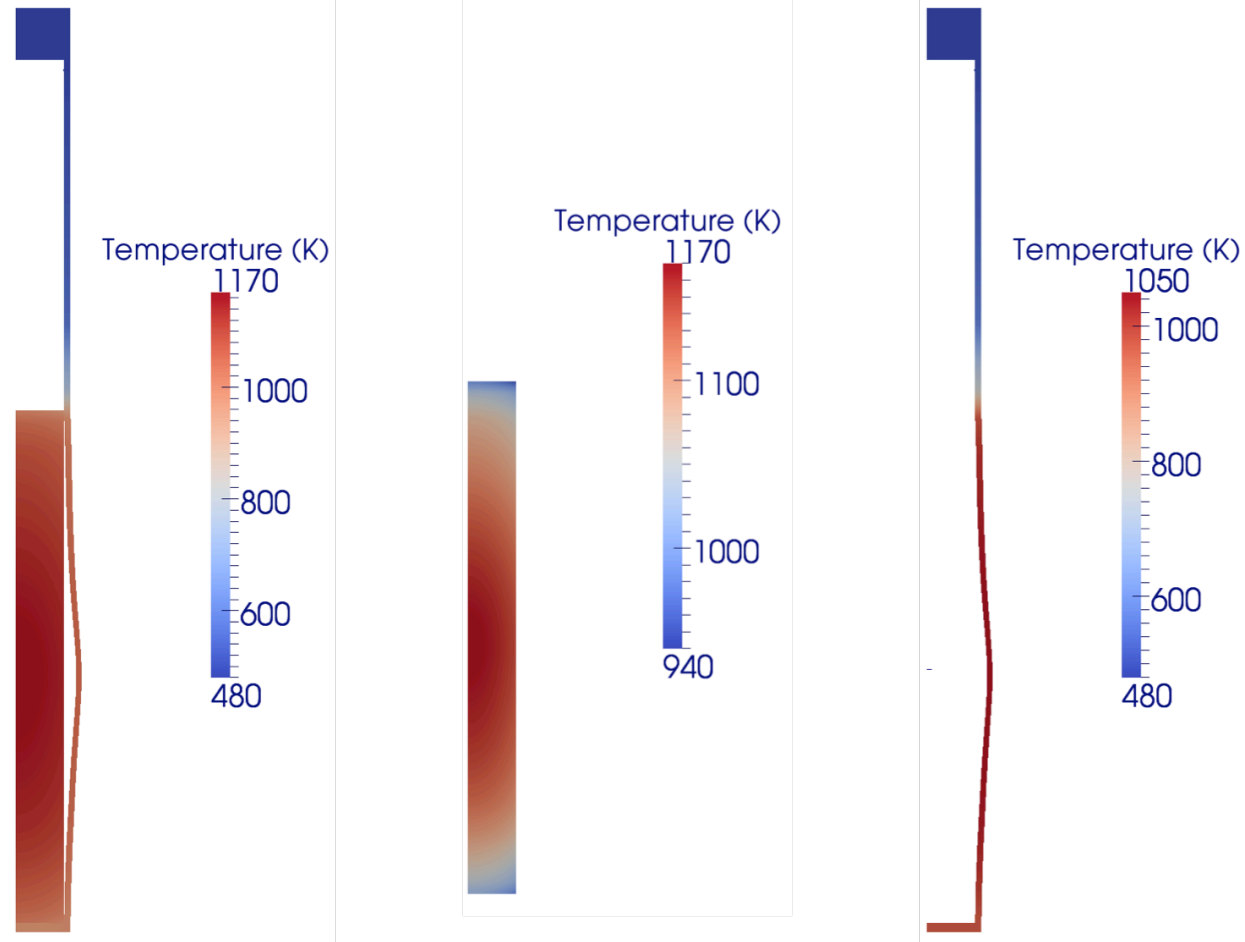

Figure 3.17. Contour plots of calculated temperature in the IFA-650.10 fuel rod at the time of cladding burst failure. Full rod (left), fuel only (center) and cladding only (right). The view is magnified 10 times in the radial direction for improved visualization.

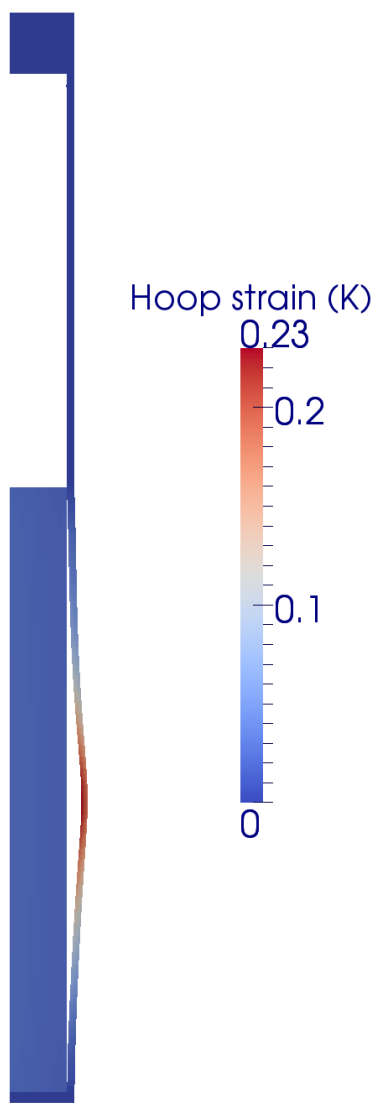

Figure 3.18. Contour plot of calculated hoop strain in the IFA-650.10 fuel rod at the time of cladding burst failure. Cladding ballooning as reproduced in the simulation is evident. The view is magnified 10 times in the radial direction for improved visualization. 
Figure 3.19 shows the axial profile of the cladding diameter at the end of the simulation compared with the experimental data from post-irradiation examinations. BISON is able to predict cladding ballooning with a physically meaningful profile and with the position of maximum strain being reasonably close to the experimental observation. However, an overprediction of cladding outward strain along the rod is observed.

As already noted in Section 3.1, the accurate prediction of maximum cladding strains reached during LOCA tests is extremely difficult. In particular, very high strain rates are attained as cladding burst is approached, which implies that the maximum strain reached in the calculation is very sensitive to the specific criterion adopted to determine the time of rod burst (i.e., the time at which the simulation ends and strain is considered), since small differences in the final time may correspond to large differences in the maximum strain. In order to potentially improve cladding strain predictions with BISON, further investigation and sensitivity analysis of the dependence of calculated strains upon the choice of the burst criterion, as well as further developments of the cladding creep model (e.g., considering anisotropic creep), are of interest in perspective.

In Figure 3.20, the calculated time evolution of rod inner pressure during the post-blowdown phases of the IFA-650.10 experiment is compared to the experimental (pressure transducer) data from Halden. BISON reproduces the experimental behavior with a good accuracy. Deviations of the calculation from the experimental data may be partly due to a discrepancy between the calculated and actual plenum temperature (which together with rod inner volume and gas content determines the plenum pressure) in consequence of the uncertainties in the temperature calculation and boundary conditions, and the assumptions in the plenum temperature

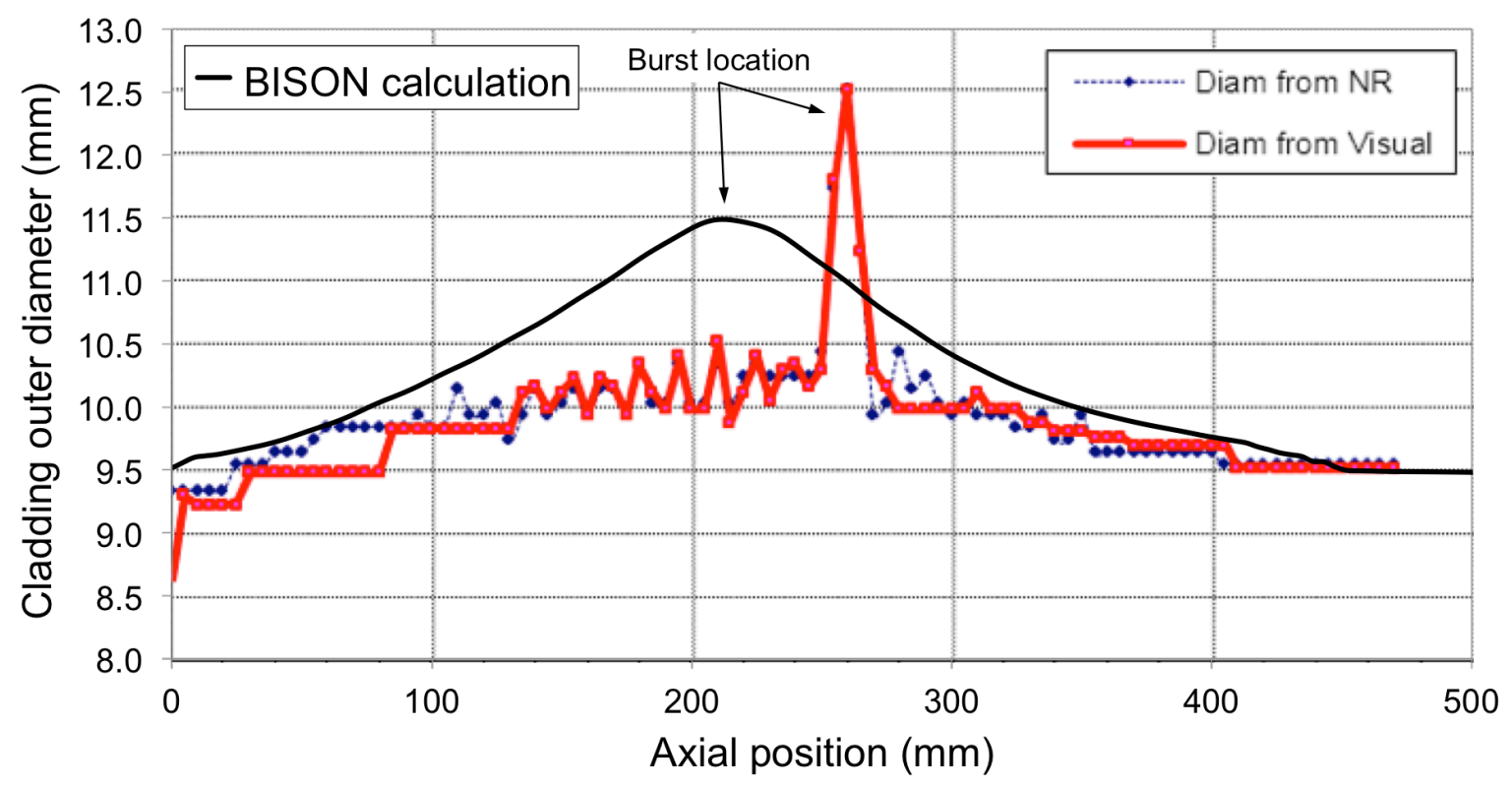

Figure 3.19. Calculated cladding outer diameter profile for IFA-650.10 at the end of the simulation compared to the Halden experimental data. 
calculation itself. ${ }^{1}$ Also, the calculated pressure as burst time is approached decreases more rapidly than experimentally observed. This is expected to be a consequence of calculated cladding outward deformation (ballooning) and the associated increase in rod inner volume being more rapid than occurred experimentally. This is confirmed by the calculated cladding diameter profile at the end of the simulation shown in Fig. 3.19. An improved treatment of cladding creep that allows for anisotropic behavior, and a refined calculation of the plenum temperature, may improve our results.

Cladding burst is predicted to occur $\sim 236$ seconds after blowdown, i.e., $\sim 13$ seconds before time observed experimentally (249 seconds after blowdown). Furthermore, BISON's prediction is conservative as the cladding is predicted to fail before it was experimentally observed.

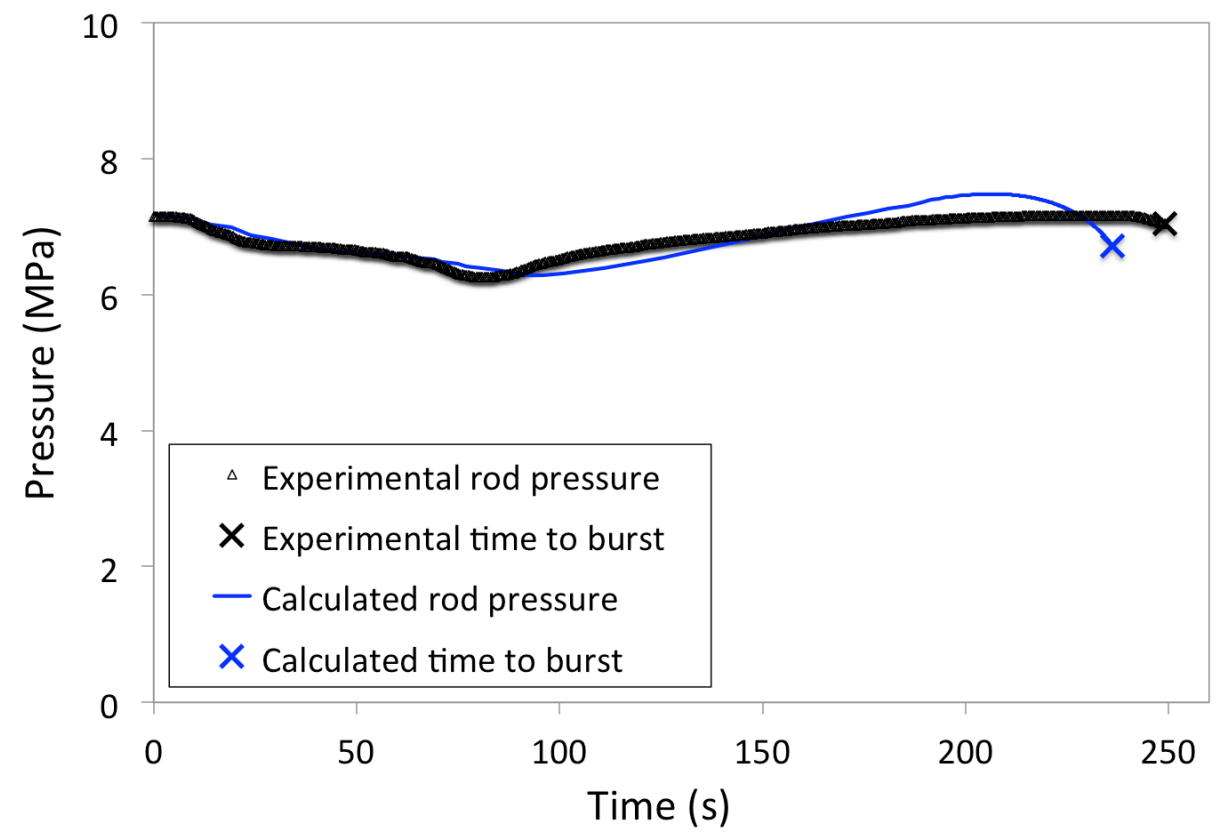

Figure 3.20. Rod inner pressure evolution during the post-blowdown phase of IFA-650.10 and time to cladding burst. BISON results are compared to the Halden experimental data.

\footnotetext{
${ }^{1}$ Plenum temperature in the BISON model of IFA- 650.10 is calculated as the average cladding inner temperature, with only the plenum height considered. Outer fuel temperature, and cladding inner temperature below the plenum (i.e., along the gap) are neglected. This assumption is deemed reasonable for an experiment such as IFA-650.10 where the plenum length and volume are high compared to standard plenum/fuel column ratios [43], so that the gas temperature is predominantly determined by the inner cladding temperature along the plenum.
} 


\section{CONCLUSIONS}

In this report we summarized the contribution of Idaho National laboratory (INL) to the FUMAC project. In line with the original research agreement, work at INL has focused on both (i) developments of INL's fuel performance code BISON for the analysis of LOCAs and (ii) simulation of selected FUMAC priority cases.

As for code developments, BISON extensions relevant to FUMAC included the incorporation into the code of the key material and behavior models required to address transient hightemperature phenomena occurring during LOCAs. In particular, models were implemented in BISON for high temperature cladding oxidation, Zircaloy solid-solid phase transformation, Zircaloy high temperature creep, cladding burst failure and axial fuel relocation.

With reference to the simulation of the FUMAC cases with BISON, as originally agreed several cases were considered, including both separate effects and integral fuel rod tests. In particular, BISON simulations were performed for the FUMAC cases (1) MTA EK tests PUZRY, (2) QUENCH L1 rods 4 and 7, (3) Halden IFA-650.2, and (4) Halden IFA-650.10. In addition, simulations of the ballooning tests REBEKA were performed and are included in this report, in view of the potential interest to the FUMAC project. This additional work included 3D simulations accounting for azimuthal temperature variations.

All of the 31 MTA EK separate effects ballooning and burst tests PUZRY were simulated with BISON. Results were reasonable in terms of cladding burst times and pressures. It was noted that discrepancies between calculations and experimental data may be partly due to anisotropic creep behavior, which is not considered in the BISON model at this time. Predictions of maximum cladding strain were less satisfactory, indicating an area of potential future improvement. It was emphasized that the accurate prediction of cladding strains reached during LOCA tests is extremely difficult, because very high strain rates are attained as cladding burst is approached, making the maximum strain reached during the calculation very sensitive to the specific burst criterion adopted and the associated uncertainties.

Additional separate effects simulations were performed for the REBEKA tests, which also pointed out a reasonable accuracy of BISON burst predictions (burst temperatures). Moreover, using BISON's 3D capability, one of the REBEKA cases was simulated in 3D, to investigate cladding response in presence of azimuthal temperature variations. Results indicated 3D effects are potentially important to accurate fuel rod analysis during LOCAs.

BISON simulations of the QUENCH L1 rods 4 and 7 confirmed a good predictive capability of the code for cladding burst temperatures and pressures, and less satisfactory predictions of cladding strains. In particular, maximum cladding hoop strains were under-predicted. As noted above, this is expected to be, to a significant extent, related to the high sensitivity of calculated maximum strains to the choice of and uncertainties in the cladding burst criterion.

Simulation of the Halden test IFA-650.2 involved determination of the thermal boundary conditions at the cladding outer wall following an approximate calculation procedure based on the Halden thermocouple data. Results were compared to experimental data of rod inner pressure evolution during the test and time to cladding burst. Both quantities were reasonably well predicted by BISON. Rod pressure was moderately over-predicted during the heat-up phase of the test, which may be ascribed to discrepancies in the calculated plenum temperature and/or evolution of fuel rod inner volume during ballooning. Calculated time to burst was $\sim 7$ seconds before the experimental one. 
Finally, we presented the BISON simulation of the Halden IFA-650.10 case. The calculation included all of the phases of the experiment from the beginning of life through the commercial base irradiation and the LOCA test. The analysis of the LOCA test was informed with the thermal boundary conditions calculated with the SOCRAT code and provided through FUMAC. The time to cladding burst failure was predicted with a good accuracy, the calculated time being within 13 seconds of the experimental one. Furthermore, the calculation was conservative, the predicted failure time being slightly earlier than experimentally observed. Also, a comparison of the cladding diameter profile at the end of the test was shown, pointing out significant discrepancies between experimental and calculated profiles. However, cladding ballooning was reproduced, with a physically meaningful profile. Finally, BISON was able to reproduce the experimental evolution of rod inner pressure during the test with a good accuracy.

The BISON results are made available to the FUMAC project as a contribution to the FUMAC benchmark exercise.

In perspective, further developments of BISON for LOCA analysis are of interest in order to enhance the code's predictive capability for LOCAs. In particular, further investigation and sensitivity analysis of the dependence of calculated maximum cladding strains upon the choice of the burst criterion is deemed useful. Also, improvements in predictions of cladding strain as well as cladding burst may be achieved by considering the anisotropic creep behavior of alpha-Zr under LOCA conditions. This will require modifying the mechanics models in the code to consider anisotropic creep strain. Another potential source of discrepancy is the 2D representation of fuel rod behavior that involves inherently $3 \mathrm{D}$ effects such as localized ballooning and burst associated with azimuthal temperature variations. As demonstrated by initial 3D simulations with BISON, 3D effects are important for LOCA analysis. Additional simulations in $3 \mathrm{D}$ with BISON to further investigate such effects are of interest in perspective.

Currently, BISON does not consider the thinning of the cladding metal wall during oxidation, which can be of importance to cladding mechanical behavior at the high oxidation rates that can be attained during LOCAs. This could be considered in a finite element code using the Extended Finite Element Method (X-FEM) to simulate a moving material interface (metal, oxide). Moreover, internal cladding oxidation is not considered at this time and is of interest for future developments. Also, a model for hydrogen production and uptake during oxidation is available in BISON and has been applied to fuel rod lifecycle simulations including spent fuel storage [47]. However, this model has not been applied yet to LOCA simulations with BISON, which represents a potential future application. Finally, coupling to thermalhydraulics system codes developed at INL is of potential interest for an improved multiphysics coupling that could result in improved boundary conditions for the BISON fuel rod calculations under accident conditions. 


\section{Acknowledgments}

This work was funded by the U.S. DOE Nuclear Energy Advanced Modeling and Simulation (NEAMS) program, the U.S. DOE Consortium for Advanced Simulation of Light Water Reactors (CASL) project, and the INL Laboratory Directed Research and Development (LDRD) program.

The submitted manuscript has been authored by a contractor of the U.S. Government under Contract DE-AC07-05ID14517. Accordingly, the U.S. Government retains a non-exclusive, royalty free license to publish or reproduce the published form of this contribution, or allow others to do so, for U.S. Government purposes.

The work fits in the framework of the Coordinated Research Project (CRP) on Analysis of Fuel Modeling under Accident Conditions (FUMAC) of the IAEA and benefited from the discussion within the CRP. Travel funding from IAEA is also acknowledged. INL participated in FUMAC under the IAEA Research Agreement No: 18464/R0.

Katalin Kulacsy (Hungarian Academy of Sciences) and Juri Stuckert (KIT) are graciously acknowledged for the discussions and information about the PUZRY and QUENCH experiments. 



\section{REFERENCES}

[1] R.L. Williamson, J.D. Hales, S. R. Novascone, M.R. Tonks, D.R. Gaston, C.J. Permann, D. Andrs, R.C. Martineau. Multidimensional multiphysics simulation of nuclear fuel behavior. Journal of Nuclear Materials, 423:149-163, 2012.

[2] R.L. Williamson, K.A. Gamble, D.M. Perez, S.R. Novascone, G. Pastore, R.J. Gardner, J.D. Hales, W. Liu, A. Mai. Validating the BISON fuel performance code to integral LWR experiments. Nuclear Engineering and Design, 301:232244, 2016.

[3] G. Pastore, S.R. Novascone, R.L. Williamson, J.D. Hales, B.W. Spencer, D.S. Stafford. Modelling of fuel behaviour during loss-of-coolant accidents using the BISON code. In Proc. of the LWR Fuel Performance Meeting, Zurich, Switzerland, September 13-17, 2015.

[4] G. Pastore, R.L. Williamson, S.R. Novascone, B.W. Spencer, J.D. Hales. Modelling of LOCA tests with the BISON fuel performance code. In Enlarged Halden Programme Group Meeting, Fornebu, Norway, May 8-13, 2016.

[5] R.L. Williamson, C.P. Folsom, G. Pastore, S. Veearaghavan. Reactivity insertion accident (RIA) capability status in the BISON fuel performance code. Technical Report CASL-X-2016-1104-000, July 2016.

[6] G. Pastore, C.P. Folsom, R.L. Williamson, J.D. Hales, L. Luzzi, D. Pizzocri, T. Barani. Modelling fission gas behaviour with the BISON fuel performance code. In Enlarged Halden Programme Group Meeting, Lillehammer, Norway, September 24-29, 2017.

[7] US DOE Participation in the FUMAC Coordinated Research Project on Fuel Performance Modeling. Proposal for research agreement between Battelle Energy Alliance and International Atomic Energy Agency for the FUMAC Coordinated Research Project, 2014.

[8] G. Pastore, D. Pizzocri, S.R. Novascone, D.M. Perez, B.W. Spencer, R.L. Williamson, P. Van Uffelen, L. Luzzi. Modelling of transient fission gas behaviour in oxide fuel and application to the BISON code. In: Enlarged Halden Programme Group Meeting, Røros, Norway, September 7-12, 2014.

[9] T. Barani, E. Bruschi, D. Pizzocri, G. Pastore, P. Van Uffelen, R.L. Williamson, L. Luzzi. Analysis of transient fission gas behaviour in oxide fuel using BISON and TRANSURANUS. Journal of Nuclear Materials, 486:96-110, 2017.

[10] G. Schanz. Recommendations and supporting information on the choice of zirconium oxidation models in severe accident codes. Technical Report FZKA 6827, SAM-COLOSS-P043, Forschungszentrum Karlsruhe, Germany, 2003.

[11] S. Leistikow, G. Schanz, H.v. Berg, A.E. Aly. Comprehensive presentation of extended Zircaloy-4/steam oxidation results 600-1600 C. In CSNI/IAEA specialists meeting on water reactor fuel safety and fission product release in offnormal and accident conditions, Risø National Laboratory, Denmark, 1983.

[12] J.V. Cathcart, R.E. Pawel, R.A. McKee, R.E. Druschel, G.J. Yurek, J.J. Campbell, S.H. Jury. Zirconium metal-water oxidation kinetics, IV. Reaction rate studies. Technical Report ORNL/NUREG-17, Oak Ridge National Laboratory, 1977.

[13] J.T. Prater and E.L. Courtright. Zircaloy-4 oxidation at 1300 to 2400 C. Technical Report NUREG/CR-4889, PNL-6166, Pacific Northwest National Laboratory, 1987.

[14] A.R. Massih. Transformation kinetics of zirconium alloys under non-isothermal conditions. Journal of Nuclear Materials, 384:330-335, 2009. 
[15] A.R. Massih and L.O. Jernkvist. Transformation kinetics of alloys under nonisothermal conditions. Modelling and Simulation in Materials Science and Engineering, 17:055002 (15pp), 2009.

[16] A.R. Massih. Evaluation of loss-of-coolant accident simulation tests with the fuel rod analysis code FRAPTRAN-1.4. Technical Report TR11-008V1, Quantum Technologies AB, 2011.

[17] P. Van Uffelen, C. Győri, A. Schubert, J. van de Laar, Z. Hoózer, G. Spykman. Extending the application range of a fuel performance code from normal operating to design basis accident conditions. Journal of Nuclear Materials, 383:137-143, 2008 .

[18] H. J. Neitzel and H. Rosinger. The development of a burst criterion for zircaloy fuel cladding under LOCA conditions. Technical Report KfK 4343, Kernforschungszentrum Karlsruhe, Germany, 1980.

[19] F. J. Erbacher, H. J. Neitzel, H. Rosinger, H. Schmidt, K. Wiehr. Burst criterion of Zircaloy fuel claddings in a loss-of-coolant accident. In Zirconium in the Nuclear Industry, Fifth Conference, ASTM STP 754, D.G. Franklin Ed., pages 271-283. American Society for Testing and Materials, 1982.

[20] M. E. Markiewicz and F.J. Erbacher. Experiments on ballooning in pressurized and transiently heated Zircaloy-4 tubes. Technical Report KfK 4343, Kernforschungszentrum Karlsruhe, Germany, 1988.

[21] V. Di Marcello, A. Schubert, J. van de Laar, P. Van Uffelen. The TRANSURANUS mechanical model for large strain analysis. Nuclear Engineering and Design, 276:19-29, 2014.

[22] G. Pastore, L. Luzzi, V. Di Marcello, and P. Van Uffelen. Physics-based modelling of fission gas swelling and release in $\mathrm{UO}_{2}$ applied to integral fuel rod analysis. Nuclear Engineering and Design, 256:75-86, 2013.

[23] G. Pastore, L.P. Swiler, J.D. Hales, S.R. Novascone, D.M. Perez, B.W. Spencer, L. Luzzi, P. Van Uffelen, R.L. Williamson. Uncertainty and sensitivity analysis of fission gas behavior in engineering-scale fuel modeling. Journal of Nuclear Materials, 465:398-408, 2015.

[24] R. M. Carroll, J. G. Morgan, R. B. Perez, O. Sisman. Fission density, burnup, and temperature effects on fission-gas release from $\mathrm{UO}_{2}$. Nuclear Science and Engineering, 38:143-155, 1969.

[25] I. J. Hastings, A. D. Smith, P. J. Fehrenbach, T. J. Carter. Fission gas release from power-ramped $\mathrm{UO}_{2}$ fuel. Journal of Nuclear Materials, 139:106-112, 1986.

[26] C. T. Walker, P. Knappik, M. Mogensen. Concerning the development of grain face bubbles and fission gas release in $\mathrm{UO}_{2}$ fuel. Journal of Nuclear Materials, 161:10-23, 1988.

[27] K. Une and S. Kashibe. Fission gas release during post irradiation annealing of BWR fuels. Journal of Nuclear Science and Technology, 27:1002-1016, 1990.

[28] SCDAP/RELAP5-3D Code Manual. Volume 4: MATPRO a library of materials properties for light-water-reactor accident analysis. Technical Report INEEL/EXT-02-00589, Idaho National Engineering and Environmental Laboratory, 2003.

[29] L. O. Jernkvist and A. Massih. Model for axial relocation of fragmented and pulverized fuel pellets in distending fuel rods and its effects on fuel rod heat load. Technical Report SSM-2015:37, 2015. 
[30] G. Pastore, R. L. Williamson, J. D. Hales. Status Report on INL Contribution to FUMAC with the BISON Code. Presentation given at the Seconds Research Coordination Meeting of the FUMAC Project, Vienna, Austria, May 30 - June 2, 2016. Available at https://nucleus.iaea.org/sites/nefw-projects/fumaccrp/SitePages/ Home.aspx.

[31] R. L. Williamson, G. Pastore, J. D. Hales. Status Report on US DOE/INL Participation in the Coordinated Research Project FUMAC. Technical report, May 2016. Submitted in support of the Second Research Coordination Meeting of the FUMAC Project.

[32] D.G. Hardy. High Temperature Expansion and Rupture Behaviour of Zircaloy Tubing. In CSNI Proceeding of the Specialist Meeting on Safety of Water Reactor Fuel Elements, Saclay, France, October 22-24 1973.

[33] C. L. Wilson et al. LOCA simulation in NRU program: Data report for the fourth materials experiment (MT-4). Technical Report NUREG/CR-3272 (PNL-4669), Pacific Northwest Laboratory, July 1983.

[34] C. L. Wilson et al. Large-break LOCA in-reactor fuel bundle materials test MT-6A. Technical Report PNL-8829, Pacific Northwest Laboratory, September 1993.

[35] R. L. Williamson, G. Pastore, K. A. Gamble, R. J. Gardner, J. Tompkins, W. Liu. Development of a LOCA Experimental Benchmark for BISON. Technical Report CASL-U-2017-1422-000, Consortium for Advanced Simulation of LWRs, 2017.

[36] Z. Hózer, C. Győri, M. Horváth, I. Nagy, L. Maróti, L. Matus, P. Windberg, J. Frecska. Ballooning Experiments with VVER Cladding. Nuclear Technology, 152:273-285, 2005.

[37] E. Perez-Feró, C. Győri, L. Matus, L. Vasáros, Z. Hózer, P. Windberg, L. Maróti, M. Horváth, I. Nagy, A. Pintér-Csordás, T. Novotny. Experimental database of E110 claddings exposed to accident conditions. Journal of Nuclear Materials, 397:48-54, 2010.

[38] K. Kulacsy. Communication within the FUMAC Project, March 2015.

[39] F.J. Erbacher, H.J. Neitzel, K. Wiehr. Technical Report KfK 4781, Kernforschungszentrum Karlsruhe, Germany, 1990.

[40] J. Stuckert, M. Grobe, C. Rossger, M. Steinbruck, M. Walter. Results of the LOCA reference bundle test QUENCH-L1 with Zircaloy-4 cladding. Technical Report KIT-SR 7651, Karlsruhe Institute for Technology, Germany, 2015.

[41] M. Ek. LOCA Testing at Halden; The Second Experiment IFA-650.2. Technical Report HWR-813, OECD Halden Reactor Project, 2005.

[42] https://nucleus.iaea.org/sites/nefw-projects/fumaccrp/SitePages/Home.aspx.

[43] A. Lavoil. LOCA Testing at Halden; The Tenth Experiment IFA-650.10. Technical Report HWR-974, OECD Halden Reactor Project, 2010.

[44] Short Information on the Results of IFA-650.10 and IFA-650.11 Calculations with SOCRAT code. Technical note, version 1. Technical report, 2016. Released to the FUMAC project. Available at https://nucleus.iaea.org/sites/nefw-projects/ fumaccrp/SitePages/Home.aspx.

[45] A. Lavoil. LOCA Experiments IFA-650.10. Technical Report EP-1650.10, OECD Halden Reactor Project, 2010.

[46] Extract from Technical Report HWR-664, OECD Halden Reactor Project. At https://nucleus.iaea.org/sites/nefw-projects/fumaccrp/SitePages/Home.aspx

[47] D.S. Stafford. Multidimensional simulations of hydrides during fuel rod lifecycle. Journal of Nuclear Materials, 466:362-372, 2015. 